\title{
REVIEW
}

\section{Death comes on two wings: a review of dipteran natural enemies of arachnids}

\author{
Jéssica P. Gillung and Christopher J. Borkent: California State Collection of Arthropods, California Department of Food \\ and Agriculture, 3294 Meadowview Road, Sacramento, CA 95832, USA; E-mail: jpg.bio@gmail.com
}

\begin{abstract}
Though the best known natural enemies of arachnids are Hymenoptera, Diptera also form an important group of arachnid enemies, attacking 31 spider families in all three suborders of Araneae, as well as members of the Acari, Amblypygi and Scorpiones. Some species of Bombyliidae, Chloropidae, Drosophilidae, Ephydridae, Phoridae and Sarcophagidae are known to attack eggs of several families of arachnids, acting as predators, parasitoids and/or parasites of egg sacs. Alternatively, members of Acroceridae and Tachinidae are internal parasitoids, attacking juvenile and/or adult spiders. One species of Sarcophagidae is reported as a predator of individual Liphistiidae (Mesothelae) spiders. We summarize the available information on all lineages of Diptera known to attack arachnids, including predators, parasites, kleptoparasites and parasitoids. A table including host records pertaining to the aforementioned dipteran families is presented. Particular emphasis is given to Acroceridae, the only lineage of Diptera known to develop exclusively on arachnids, and one of the most significant groups of natural enemies of spiders.
\end{abstract}

Keywords: Araneae, parasitoid, spider egg sacs, scorpions, amblypygids

\section{TABLE OF CONTENTS}

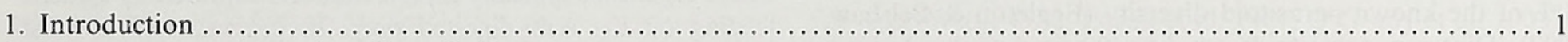

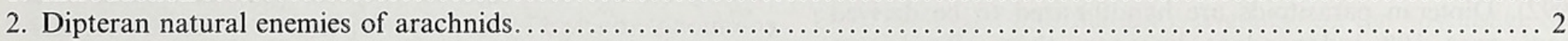

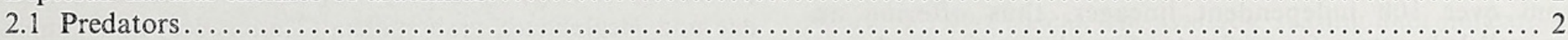

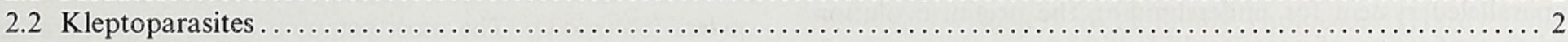

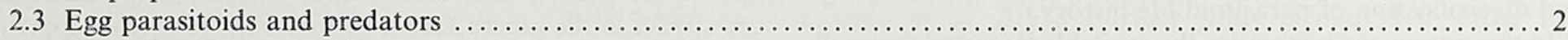

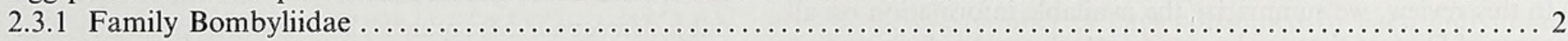

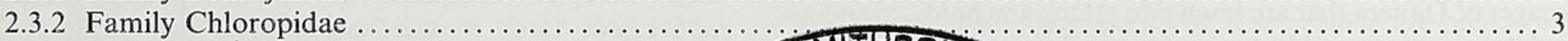

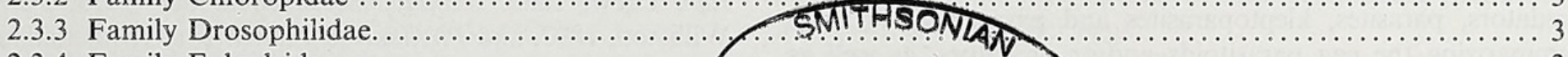

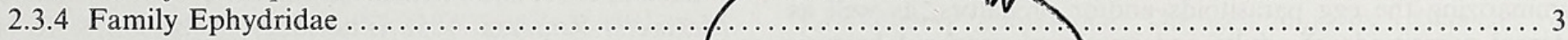

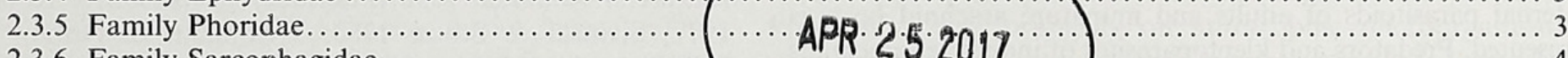

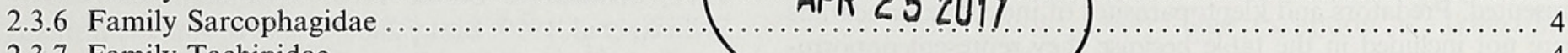

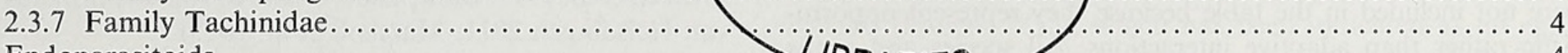

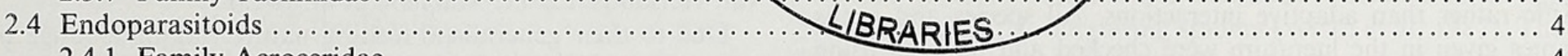

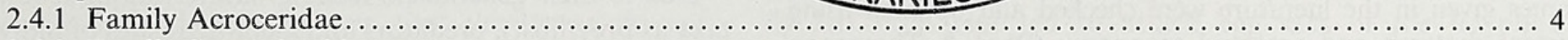

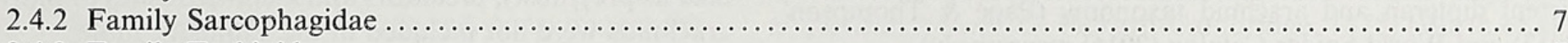

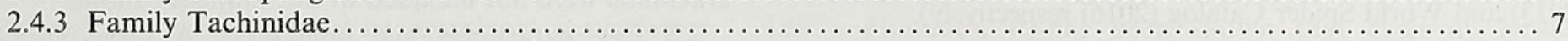

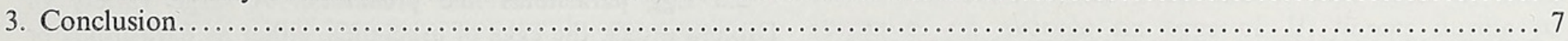

\section{INTRODUCTION}

Some of the best known natural enemies of arachnids belong to the order Hymenoptera, with species either feeding on the individual arachnid, or on its eggs. Several groups of Hymenoptera develop on arachnid hosts, including wasps in the families Diapriidae, Eulophidae, Eupelmidae, Eurytomidae, Ichneumonidae, Platygastridae sensu lato, Pompilidae, Pteromalidae and Sphecidae (Fitton et al. 1987; Noyes 2016). Hymenopteran parasitoids and predators exhibit a breadth of life strategies, including species that feed in cocooned spider egg masses, endoparasitoids that develop individually within eggs, external koinobiont parasitoids of mobile spiders, and idiobionts that paralyze one or more spiders as prey (Austin 1985; Gauld \& Dubois 2006).

Species in the order Diptera are also significant natural enemies of arachnids. As opposed to the large taxonomic diversity of hymenopteran arachnid enemies, dipteran parasites, parasitoids and predators of arachnids are restricted to fewer families and vary considerably in their mode of action. All species of Acroceridae and a single species of Tachinidae are internal parasitoids of juvenile or adult spiders, while a few members of the Chloropidae, Drosophilidae, Ephydridae, Phoridae and Sarcophagidae are known to attack egg sacs of several arachnids, acting as parasitoids and/or predators of arachnid eggs (Clausen 1940; Vincent 1985; Schlinger 1987; Marshall 2012). One species of Sarcophagidae is a predator of Mesothelae spiders, attacking both adult and immature prey (Schwendinger \& Pape 2000).

Although a clear cut distinction between predator and parasite is not always possible in nature, the three extremes of the spectrum (i.e., true parasite vs. true predator vs. true parasitoid) can be easily distinguished. Predators are organ- 
isms that consume one or multiple prey during their lifetime, thus establishing a relationship that positively affects the predator and negatively affects the prey. Parasites, in general, live on/in the body of the organism they feed upon, and in contrast to predators, only take resources from one host, which they tend not to kill. Parasitoids, like parasites, only attack one host, but they enter or attach to their host, feed upon it, and ultimately kill it. Thus, the essential difference between parasites and parasitoids is that parasitoids always kill their hosts, while parasites tend to not kill their hosts. Additionally, the main difference between predators and parasitoids relates to the number of individuals attacked; predators usually kill multiple prey during their lifetime, while parasitoids only kill one host (Price 1980).

Parasitoids are found in five orders of holometabolous insects: Hymenoptera, Diptera, Coleoptera, Lepidoptera, and Neuroptera. Hymenopteran parasitoids account for nearly $78 \%$ of the estimated number of species, and consequently have served as models for nearly all recent research on insect parasitoids (Feener \& Brown 1997). However, parasitoids in the Hymenoptera represent a single evolutionary lineage in contrast to the hundreds of parasitoid lineages in Diptera, Coleoptera, and other orders (Eggleton \& Belshaw 1992). There are over 16,000 species of described dipteran parasitoids, which are distributed in 21 families and represent about $20 \%$ of the known parasitoid diversity (Eggleton \& Belshaw 1992). Dipteran parasitoids are hypothesized to be derived from over 100 independent lineages, thus offering an unparalleled system for understanding the origin, evolution and diversification of parasitoid life history.

In this review, we summarize the available information on all lineages of Diptera that are known to attack arachnids, including predators, parasites, kleptoparasites and parasitoids. A table summarizing the egg parasitoids and/or predators, as well as internal parasitoids of adults and immature arachnid hosts is presented. Predators and kleptoparasites of individual arachnids were not included in the table because they represent opportunistic rather than adaptive interactions. All species and genus names given in the literature were checked and updated using current dipteran and arachnid taxonomy (Pape \& Thompson (2013) and World Spider Catalog (2016) respectiveiy).

\section{DIPTERAN NATURAL ENEMIES OF ARACHNIDS}

2.1 Predators.-Species of Asilidae are among the best known dipteran predators, but it has been indicated that arachnids do not comprise a significant portion of robber fly diet, making up less than $2 \%$ of all their consumed prey (Dennis et al. 2012). Robber flies are found worldwide and may occasionally take spiders as prey, especially species in the asilid genera Daspletis Loew, 1858, Efferia Coquillett, 1893, Euscelidia Westwood, 1850, Holopogon Loew, 1847, Laphystia Loew, 1847, Psilonyx Aldrich, 1923 and Stichopogon Loew, 1847 (Dennis et al. 2012). Robber flies are recorded to prey on spiders belonging to the suborder Araneomorphae, mainly in the families Agelenidae, Araneidae, Clubionidae, Lycosidae, Salticidae and Theridiidae, among others (Dennis et al. 2012). This suborder comprises species that are generally readily visible, including orbweavers, crab spiders and jumping spiders. In contrast, spiders in the other two suborders of Araneae (Mygalomorphae and Mesothelae) generally have reclusive habits (such as trapdoor and funnel-web spiders) or are highly mobile. There are also smaller dipterans that play a role as predators of small arachnids. For instance, larvae of species in the gall midge genus Feltiella Rubsaamen, 1910 (Cecidomyiidae) are specialized predators of all spider mite life stages (Acarina: Tetranychidae), as are many members of the gall midge tribe Lestodiplosini (Gagné 1995). One species of Ceratopogonidae, Forcipomyia araneivora Clastrier \& Legrand, 1991, also plays a role as an enemy of spiders by feeding directly on their haemolymph (Clastrier \& Legrand 1991). Other common dipteran predators (e.g., Muscidae and several families in Empidoidea) may take arachnids as prey, but no records of these were found.

2.2 Kleptoparasites.-Kleptoparasitism is a form of competition that involves the stealing of a portion of already acquired food items, and is one of the most common types of exploitation between animals. Kleptoparasites steal food that is either already in a predator's possession, or which the predator has already spent energy pursuing and capture by the predator is imminent. In this interaction, the kleptoparasite is benefited and the host may potentially be negatively affected by the loss of food resources (Brockmann \& Barnard 1979; Barnard 1984). Some fly species in the families Cecidomyiidae, Ceratopogonidae, Chloropidae, Dolichopodidae, Lonchaeidae, Milichiidae and Phoridae are known kleptoparasites of spiders. These organisms typically exploit cadavers captured by spiders, feeding on the semi-digested prey. In general, spiders most commonly attacked by kleptoparasites are orbweavers (Araneidae and Nephilidae), crab spiders (Thomisidae) and jumping spiders (Salticidae). The most common spider kleptoparasites are species in the genera Didactylomyia Felt, 1911 (Cecidomyiidae), Microphor Macquart, 1827 (Dolichopodidae), Desmometopa Loew, 1866, Neophyllomyza Melander, 1913, Paramyia Williston, 1897, and Phyllomyza Fallen, 1810 (Milichiidae) and Megaselia Rondani, 1856 (Phoridae) (Robinson \& Robinson 1977; Sivinski \& Stowe 1980; Weinmann \& Disney 1997; Sivinski et al. 1999; Brake 2000; Brake \& von Tschirnhaus 2010; van Helsdingen 2011; Marshall et al. 2015).

Due to their generalistic feeding nature (taking a range of taxa as prey/host), predators and kleptoparasites of individual arachnids were not included in the summary table.

2.3 Egg parasitoids and predators. - A large variety of Diptera attack the eggs of arachnids, either as parasitoids or predators; it is often difficult to distinguish between these alternatives due to the limited number of natural history and rearing observations. Therefore, we have decided not to distinguish between these modes, in order to avoid making incorrect assumptions about poorly known dipteran-spider interactions. Each dipteran family is discussed in more detail below.

2.3.1 Family Bombyliidae: Bombyliidae is a large group of Diptera commonly known as bee flies. Despite the great number of species (over 5,000 valid species), the habits of most immature stages $(>80 \%)$ are still poorly understood (Yeates \& Greathead 1997). Known species are mostly ecto- or endoparasitoids on the larvae and/or pupae of other insects, mainly in the Lepidoptera, Hymenoptera, Coleoptera, Diptera and Neuroptera. The only known exception is Petrorossia feti Zaitsev \& Charykuliev, 1981, which develops as a parasitoid or predator on egg sacs of oecobiid spiders (Zaitsev \& Charykuliev 1981; Yeates \& Greathead 1997). It is unknown 

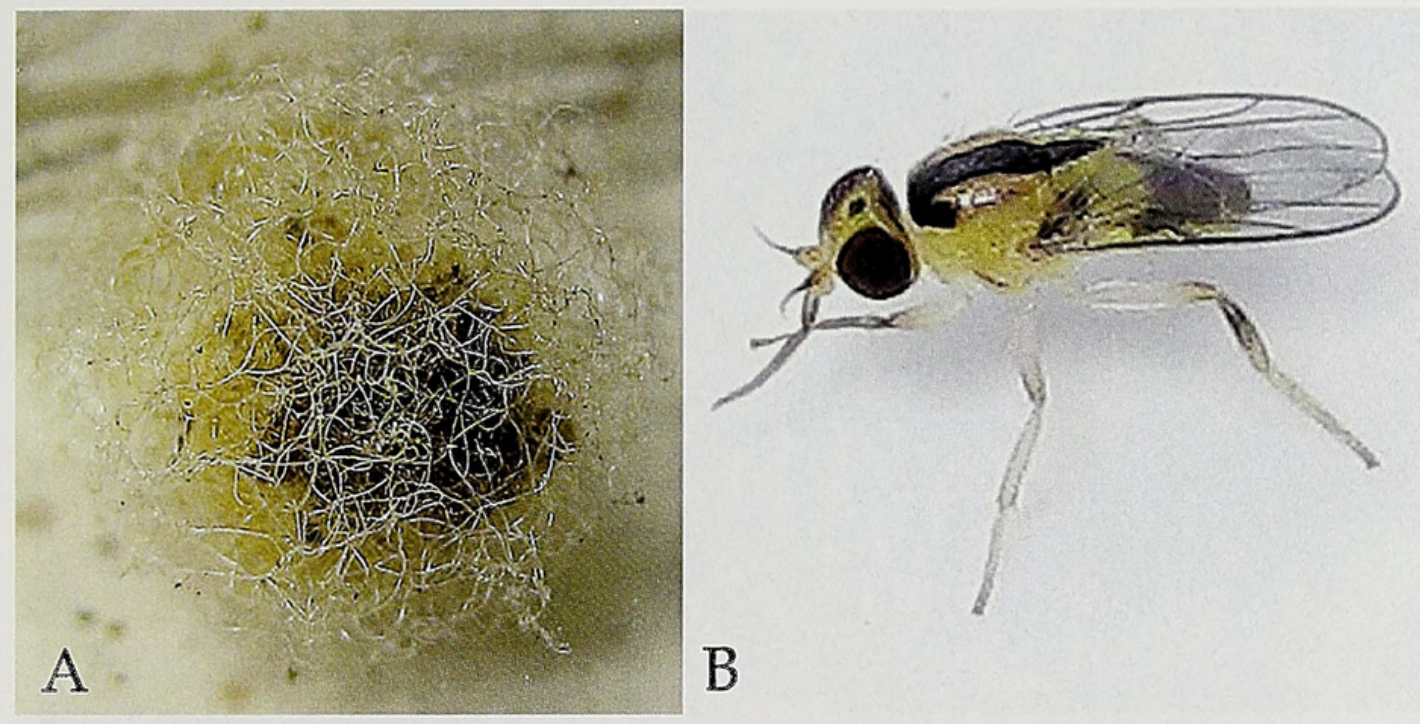

B

Figure 1.-Chloropidae development on spider egg sac. A. Egg mass of Mimetus puritanus Chamberlin, 1923 containing larvae of Pseudogaurax anchora (Loew, 1866). B. Adult P. anchora. Photos: John R. Maxwell.

whether or not this species has a specific host association with these spiders (Zaitzev \& Charykuliev 1981).

2.3.2 Family Chloropidae: Several species of Chloropidae are known to be either parasitoids or predators of arachnid eggs. Genera of chloropids that have been reported as arachnid egg predators include Conioscinella Duda, 1929, Gaurax Loew, 1863, Oscinella Becker, 1909, Oscinisoma Lioy, 1864, Pseudogaurax Malloch, 1915, Siphonella Macquart, 1835, and Tricimba Lioy, 1864 (Barnes et al. 1992). Eason et al. (1967), Rollard (1984) and Barnes et al. (1992) provide a review of host records for several groups of Diptera and Hymenoptera, including chloropids. Several species of Gaurax and Pseudogaurax are suggested to be parasitoids of arachnid eggs (including amblypygids (Viquez \& de Armas 2009; Chapin \& Hebet 2016)) and have been raised from a variety of host eggs (see details in Table I) (Figs. 1 \& 2). However, very little is known about the life histories of most of these species, and there is an indication that the larvae do not complete their development on a single egg, consuming a few or several eggs and, as such, must be considered as predators (Barnes et al. 1992). Some species are apparently opportunists, utilizing a variety of host species or even hosts of different orders, including lepidopteran cocoons and mantid oothecae. Pseudogaurax signatus (Loew, 1876) has been suggested as a biocontrol agent of black widow spiders, Latrodectus Walckenaer, 1805 , since many specimens have been reared from egg sacs of this spider (Barnes et al. 1992; Vetter et al. 2012), with infestation rates reported to range from $6 \%$ (Vetter et al. 2012) to $40 \%$, and estimated spider mortality $>90 \%$ (Barnes et al. 1992). Female $P$. signatus flies lay the eggs on the surface of the host egg sac. Once hatched, larvae enter the egg sacs and consume multiple eggs, developing inside the sac.

2.3.3 Family Drosophilidae: Most species of Drosophilidae feed on decaying fruit and fungal material, as well as on fresh sap and nectar from flowers. Species in the subgenus Titanochaeta Knab, 1914 (genus Scaptomyza Hardy, 1850), however, differ from all other drosophilids in being parasitoids (or predators) of spider eggs. Unlike the remaining species of Scaptomyza, species in the subgenus Titanochaeta exhibit a slender, sharply pointed, stylet-like ovipositor, which is likely an adaptation to a lifestyle as a spider egg sac parasitoid or predator (O'Grady et al. 2003). The group is endemic to Hawaii and comprises 11 species known to develop on spider egg sacs (Wirth 1952; Eason et al. 1967; O'Grady et al. 2003). Little biological information is available on the host usage of species of Titanochaeta, but available rearing data indicate a preference for spiders in the family Thomisidae (Hardy 1965; Lapoint et al. 2013). Further study is needed in order to investigate the number of eggs consumed by each individual, thus confirming whether species of Titanochaeta are predators or parasitoids of spider eggs.

2.3.4 Family Ephydridae: The vast majority of species of Ephydridae feed primarily on autotrophic microorganisms such as algae (Foote 1984). One exception is Trimerina madizans (Fallen, 1813), which has been indicated as an egg parasitoid of marsh-inhabiting spiders (Wirth et al. 1987). The fly has been reported to attack egg sacs of the linyphiid Hypselistes florens (O. Pickard-Cambridge, 1875) (Wirth et al. 1987). However, it has been shown that each fly larva consumes on average six eggs of $H$. florens during its development (Foote 1984); in this case, the species is better regarded as a predator rather than a parasitoid.

2.3.5 Family Phoridae: Although most species of Phoridae associated with arachnids are merely saprophagous, some are known to be associated with living rather than dead arachnids. Most of these are predators of arachnid eggs. However, specimens of Apocephalus borealis Brues, 1924 have been reared from black widow egg sacs, Latrodectus mactans (Fabricius, 1775), (Araneae: Theridiidae), and this has been suggested to be a case of true parasitism, although further research is needed for proper confirmation (Disney 1994). Several species in Megaselia Rondani, 1856 and Phalacrotophora Enderlein, 1912 (Fig. 3) are known to prey on eggs of spiders in the families Araneidae (Argiope Audouin, 1826, Gasteracantha Sundevall, 1833, and Larinioides Caporiacco, 1934), Linyphiidae (Pityohyphantes Simon, 1929), Salticidae (Phidippus C. L. Koch, 1846), Tetragnathidae (Meta C. L. Koch, 1836) and Theridiidae (Enoplognatha Pavesi, 1880, 


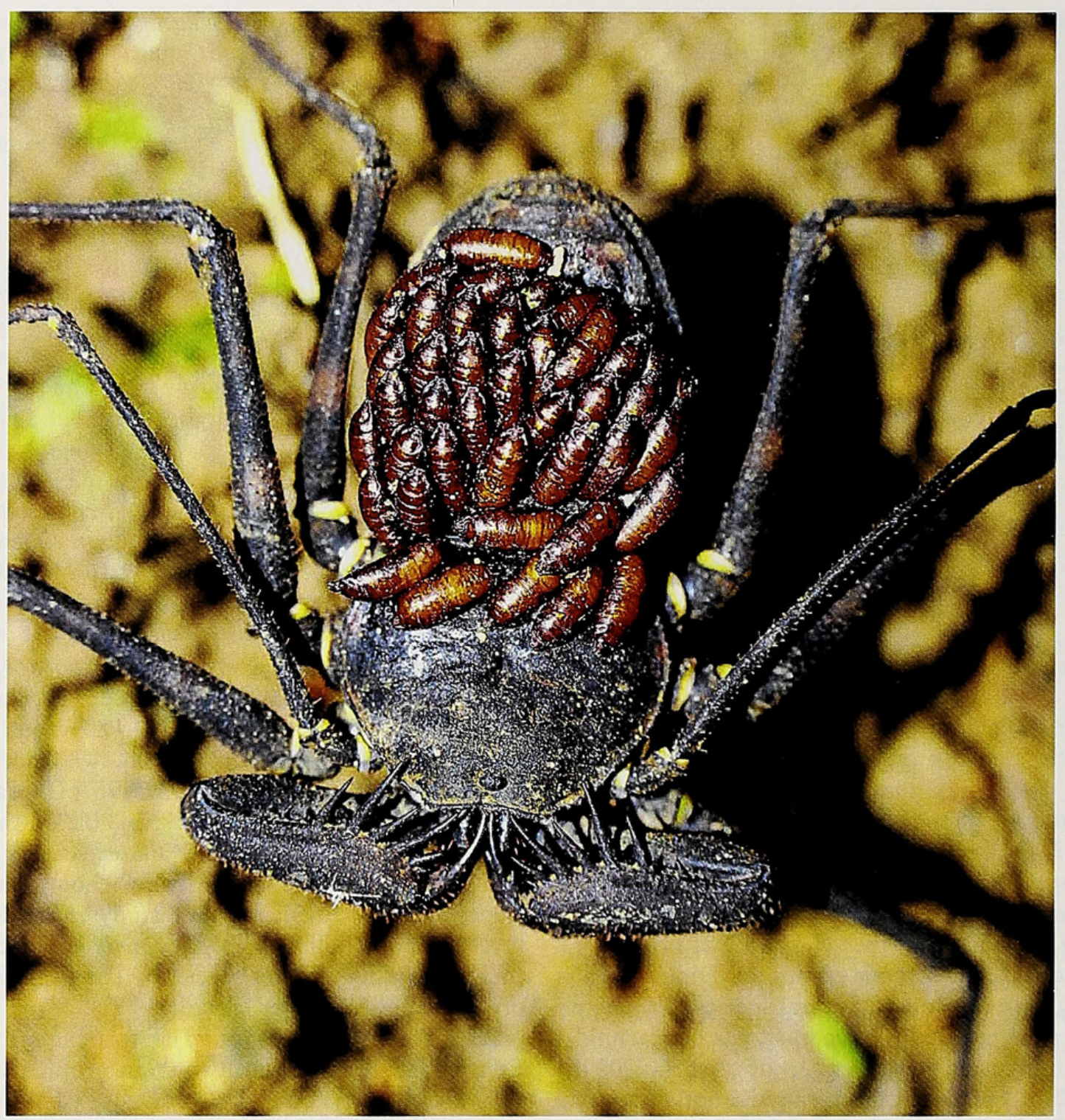

Figure 2.-Amblypigid female carrying pupae of Pseudogaurax (Diptera: Chloropidae) that were egg parasitoids of her egg case as larvae. Photographed at the Soltis Center, San Juan de Peñas Blancas, San Ramón, Costa Rica, by Kristie Reddick and Jessica Honaker.

Latrodectus and Robertus O. Pickard-Cambridge, 1879) (Disney \& Evans 1980; Disney 1982, 1994, 1999).

2.3.6 Family Sarcophagidae: Sarcophagidae is a large family of Diptera with species exhibiting a breadth of habits and life histories. Most species are generalist scavengers and insect predators, while some species are kleptoparasites of solitary bees and wasps (Pape et al. 2012). Several species in the genus Sarcophaga Meigen, 1826 are spider egg parasitoids or predators (e.g., Eason et al. 1967; Auten 1925; Prakash \& Pandian 1977; Austin 1985; Souza Lopes 1985; Cantrell 1986; Hieber \& Uetz 1990). However, as in the dipteran families mentioned above, more research is needed in order to confirm whether these species are parasitoids or predators. Schwendinger \& Pape (2000) reported an unusual case of a predatory species of Metopia Meigen, 1803, a genus that mostly comprises kleptoparasites in nests of solitary aculeate Hymenoptera (Pape 1986). Larvae of Metopia sinensis Pape, 1986 apparently kill the spider host and complete most of their larval life on the carcass, behaving more like predators rather than parasites (following Price 1980).
2.3.7 Family Tachinidae: As discussed below, tachinids are well known as parasitoids of a range of insects. However, there is only a single record of a tachinid attacking spider eggs, a specimen of Tachina Meigen, 1803 which had been reared from egg sacs of the araneid Larinioides cornutus (Clerck, 1757) (Bertkau 1880).

2.4 Endoparasitoids. - The majority of dipteran endoparasitoids of arachnids are species of the family Acroceridae, which have exclusively been reared from a variety of spider families. There are also a few examples from other Diptera families (Phoridae, Sarcophagidae and Tachinidae). The specific details of the behaviors in each case are given below.

2.4.1 Family Acroceridae: Species of Acroceridae are commonly called spider flies due to their tight relationship with spiders as internal parasitoids. They are sometimes also referred to as small-headed flies in reference to the disproportionately small heads of some species. Spider flies represent a morphologically heterogeneous assemblage of lineages, currently classified into three subfamilies (Acrocerinae, Panopinae and Philopotinae), 55 genera and approximately 530 


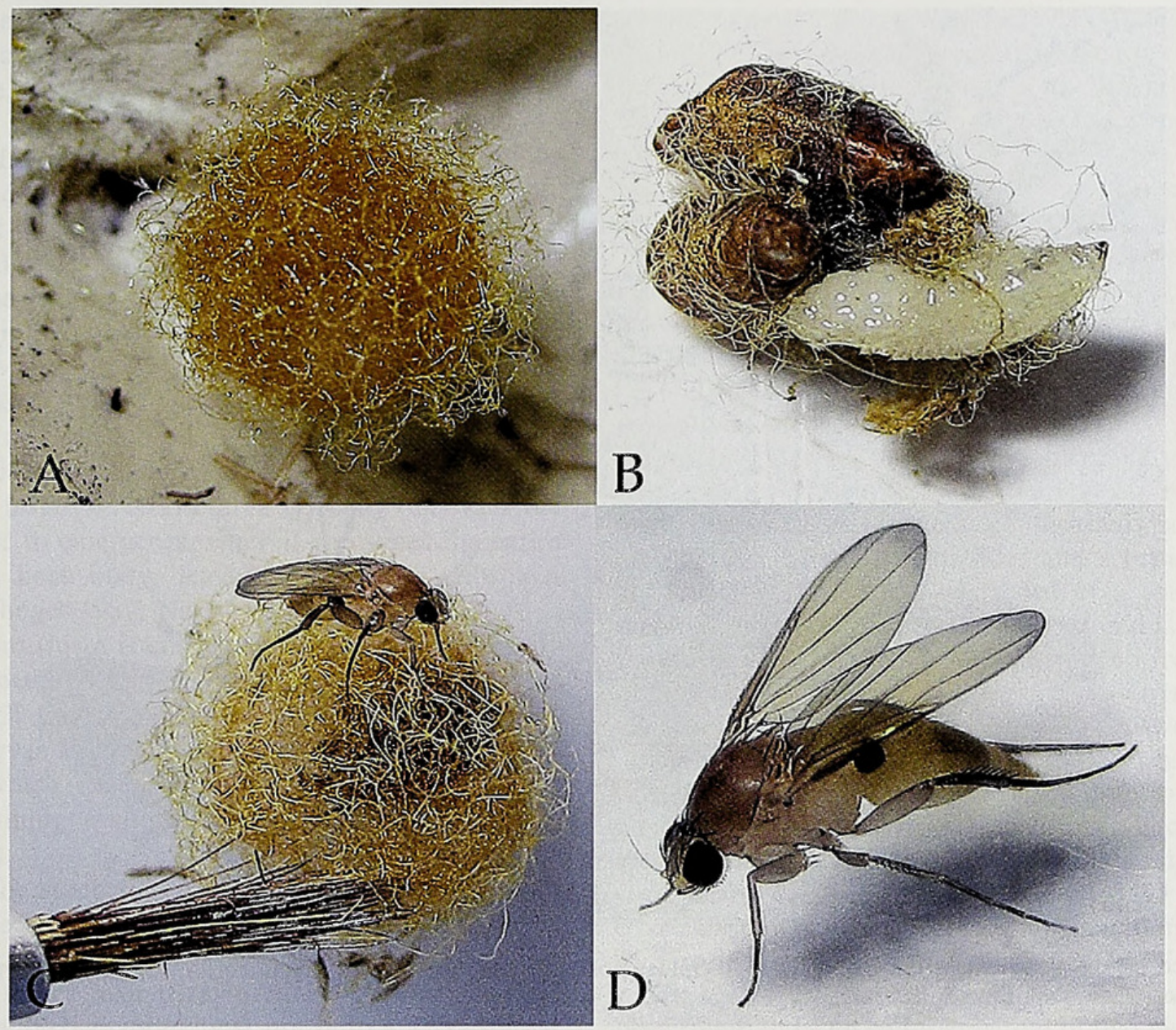

Figure 3.-Phoridae development on spider egg sac. A. Egg mass of Mimetus puritanus Chamberlin, 1923. B. Pupae and larva of Phalacrotophora epeirae (Brues, 1902). C. Spider egg mass, pupae and adult of $P$. epeirae. D. Adult $P$. epeirae, oblique view. Photos: John R. Maxwell.

species (Schlinger et al. 2013). Adults are morphologically very distinctive, usually with inflated or hunchbacked bodies, and occasionally with metallic coloration. Several species have long, modified mouthparts for nectar feeding and are considered important pollinators (e.g., Goldblatt et al. 1997; Potgieter et al. 1999; Pujol-Luz 2004; Carvalho \& Machado 2006; Borkent \& Schlinger 2008).

Spider fly larvae attack spiders in the Mygalomorphae (Fig. 4) and Araneomorphae. The only exception to the exclusive endoparasitic mode in Acroceridae is found in the Chilean genus Carvalhoa Koçak \& Kemal, 2013 (= Sphaerops Philippi, 1865), which is reported to remain ectoparasitic on its host spider for at least three weeks (Schlinger 1987). It is still unknown whether the entire lifecycle is spent as an ectoparasite, but Schlinger (1987) reports this ectoparasitic behavior in multiple rearings of later instars of the species.

Records of acrocerids parasitizing Acari are extremely rare (Sferra 1986; Kerr \& Winterton 2008), and no spider fly planidium has been reared from living mites to confirm, or identify, the acrocerid species in question. It is likely that the presence of acrocerid first instar larvae in mites is merely accidental parasitism, and the larvae probably do not develop further due to the small size of the mites relative to known acrocerid adults. Acrocerids are known to attack juvenile spiders, but even the smallest known spider hosts are several times larger than mites found in association with acrocerid planidia (Sferra 1986; Kerr \& Winterton 2008). In the cases of mite parasitism, it is likely that the planidium is simply indiscriminately adhering to arachnids in an attempt to find a suitable host. In this case, the association between Acroceridae and Acari would not represent a true host-parasitoid relationship.

Generally, adult spider fly females scatter eggs during flight or oviposit large numbers of microtype eggs on twigs, branches or foliage (Schlinger 1987). In most species, oviposition seems to be entirely independent of the presence of a host, though naturally the flies occur in a habitat favorable to spider populations. Females of Eulonchus Gerstaecker, 1856 are reported to be attracted to burrows of trapdoor spiders in the genus Antrodiaetus Ausserer, 1871. Coyle (1971) reports a case in which, as trapdoor spider burrows were being excavated, a large number of spider fiies quickly approached, hovering close to the ground and landing near closed burrow entrances, apparently attracted by some chemical released during the excavation process.

The first-instar acrocerid is a free-living planidium, which actively seeks out a spider host by crawling, looping or jumping, with the aid of well-developed setae, spines and a caudal suction disk (Schlinger 1981). Planidia in the genus Acrocera Meigen, 1803, however, differ from all other spider 


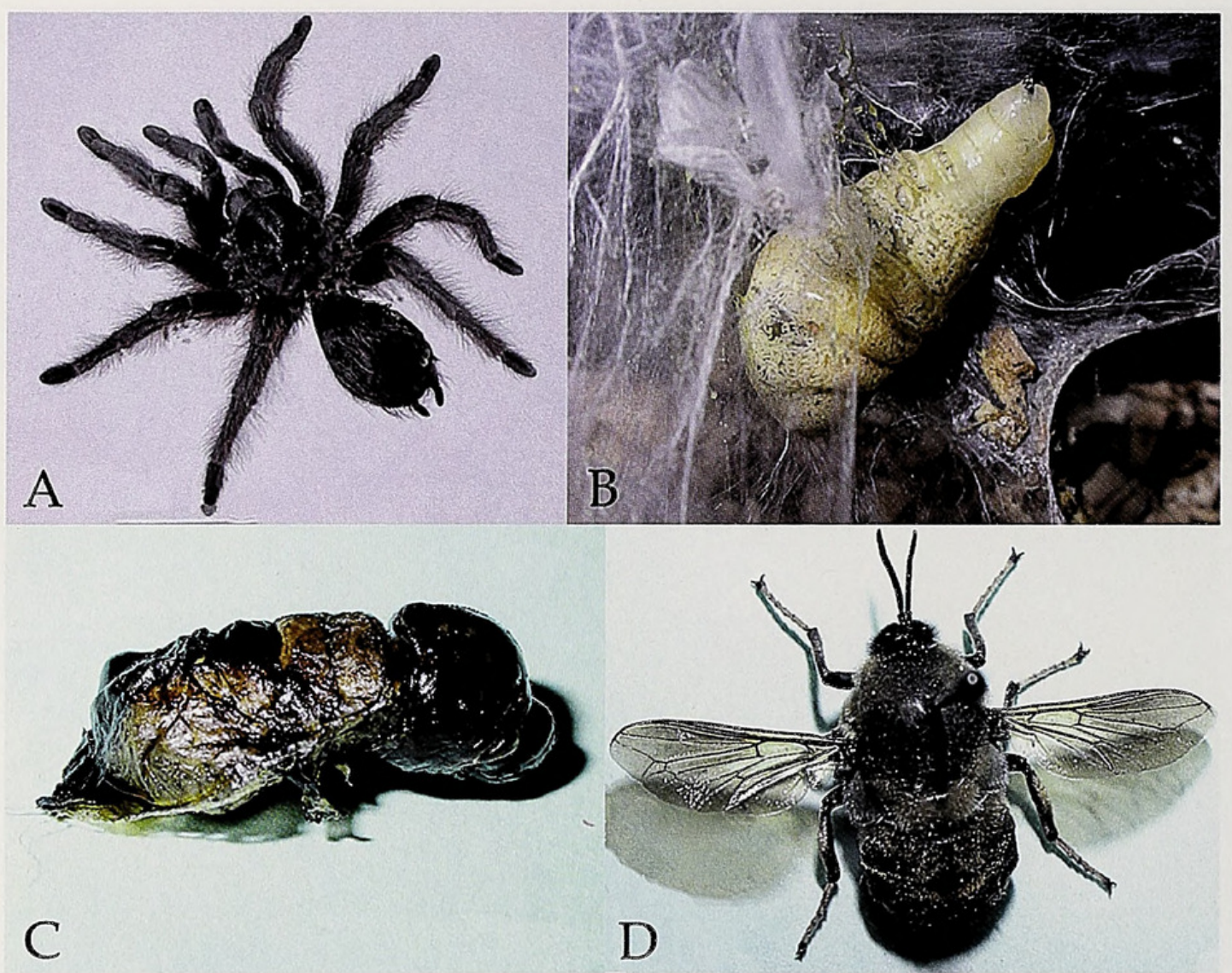

Figure 4.-Acroceridae development in the spider host. A. Spider host, Aphonopelma duplex (Chamberlin, 1925). B. Fourth instar larva of Ocnaea sp. C. Pupa of Ocnaea sp. D. Adult Ocnaea sp. Photos: Dr. A. Alagon.

flies in having a poorly sclerotized body and an abdomen with numerous annulations obscuring the regular body segmentation, as well as the absence of setal pile characteristic of acrocerid planidia (Schlinger 1981). Acrocera also exhibits a unique mode of entering its host, in which the first-instar larva injects itself into the spider. Planidia of Acrocera orbicula (Fabricius, 1787) have been observed entering a wolf spider through this sophisticated mode, in which first-instars firmly attach themselves to the host's cuticle by the mouthparts, presumably feeding externally for about a week (Overgaard Nielsen et al. 1999; Toft et al. 2012). Rather than entering the spider, the first-instar subsequently molts and the small, flexible, and glabrous second-instar is injected directly into the spider via the wound, leaving the exuvia of the ectoparasitic first-instar covering the site of infection. This mode of host invasion may reduce physical damage to the host in the initial phase of endoparasitism, thus enhancing parasitoid survival (Overgaard Nielsen et al. 1999). More research is needed in order to investigate whether this mechanism for infection is also present in other Acroceridae lineages.

Most host records for Acroceridae involve rearing of mature larvae from parasitized spiders, and observations of infestation of the host by the planidium stage are rare. The planidium presumably enters the host directly through the cuticle of the cephalothorax, opisthosoma or leg joints (Schlinger 1981, 1987; Nartshuk 1997), but these apparently have not been verified by direct observations. Acrocerid larvae eventually locate themselves in the opisthosoma and attach their posterior spiracle to the spider's book lung for respiration (Schlinger 1981, 1987). Schlinger (1987) proposed that upon entering the host, the larva may enter a state of diapause that can last for several months (Acrocerinae) up to 10 years (Panopinae), and, upon cessation of diapause, the actively feeding larva completes its life cycle relatively quickly (days to weeks), undergoing up to four instars. McQueen (1983) observed wolf spiders in the genus Geolycosa Montgomery, 1904 parasitized by Pterodontia Gray, 1832 spider flies and stated that infected spiders carry the larvae for approximately a year until they are entirely consumed by the parasitoid. However, further evidence is needed to support the hypothesis of diapause within the host and to verify the actual number of larval instars in Acroceridae, because the typical number of instars in lower brachyceran flies is three.

Many parasites and parasitoids have evolved remarkable strategies to manipulate the behavior of their hosts in order to promote their own survival and reproduction. These behavioral manipulations may include alterations in phototaxis, locomotion, foraging and defensive behaviors (Moore 2002). Most spider hosts of acrocerids, however, do not exhibit any obvious external indication of parasitism, although there are reports of enlargement of the spider's opisthosoma due to the presence of spider fly larvae (e.g., Lamore 1960; Barraclough \& Croucamp 1997). It has been repeatedly reported that the host displays some abnormal, agitated behavior during the final stages of parasitism, in which the spiders walk aimlessly and incessantly scratch the lateral portions of the opisthosoma with the legs, presumably where the parasitoid is located (Cady et al. 1993; Barneche et al. 2013). In some species, 
especially in the Mygalomorphae, the emergence of the parasite seems to correspond with the premolting behavior of the spider (e.g., Montgomery 1903; Cady et al. 1993; Barneche et al. 2013). However, a causative relationship between the production of the premolting web and parasitoid emergence can only be speculative at this time, but the presence of hook-like processes on the head and/or abdomen of the spider fly larva, which are supposedly used as attachment to the web, suggests that this is not coincidental. Lamore (1960) reported an example in which a larva of Ogcodes dispar (Macquart, 1855) parasitizing a basilica spider, Mecynogea lemniscata (Walckenaer, 1841), hung itself on the spider web while also hanging on to the spider on one end for a while, then released its hold on the exoskeleton in order to pupate. The last instar acrocerid larva typically only kills the host shortly prior to emergence, when it consumes the entire contents of the host body, leaving an empty, unbroken exoskeleton (Schlinger 1987; Nartshuk 1997).

While super-parasitism is common in early instars, usually only a single acrocerid adult ultimately emerges from its host (Cady et al. 1993; Overgaard Nielsen et al. 1999). Multiple emergences of larvae from a single host are more likely found in Panopinae, which attack large Mygalomorphae spiders capable of sustaining multiple parasitoids (Schlinger 1987; Cady et al. 1993).

Host records for acrocerids parasitizing spiders are known for at least 60 species, recorded from about 25 spider families (Schlinger 1987). Schlinger (1987, table 24) presented an extensive list of spider taxa parasitized by acrocerids, while Winterton et al. (2007) mapped host use onto a DNA-based phylogeny of the family. These studies clearly demonstrate that Panopinae are host-specific to Mygalomorphae spiders (Fig. 4), while Acrocerinae and Philopotinae are host-specific to the Araneomorphae. Only the genera Acrocera and Carvalhoa have been reared from Haplogynae spiders, while Philopotinae and all remaining Acrocerinae have only been recorded to attack Entelegynae. The cosmopolitan genera Acrocera, Ogcodes Latreille, 1797 and Pterodontia have been reared from numerous hosts in multiple spider families, but most geographically restricted and species-poor genera tend to be more host-specific, generally attacking only a single spider family (Schlinger 1987). Strict host specificity between particular acrocerid and spider species seems to be rare, but some trends are evident where host preference generally follows spider guilds instead of lineages (Schlinger 1987; Cady et al. 1993). Spiders most susceptible to parasitism by Acroceridae belong to guilds of cursorial or fossorial species (e.g., Mygalomorphae: Antrodiaetidae, Ctenizidae, Migidae, Theraphosidae; Araneomorphae: Anyphaenidae, Clubionidae, Lycosidae, Salticidae, Thomisidae), or those that occupy sac, tangle or funnel-like web retreats that are close to the ground, have webs with many connections to vegetation, or visit surrounding vegetation or substrate frequently (e.g., Agelenidae, Amaurobiidae, some Araneidae, Dipluridae, Segestriidae) (Schlinger 1987; Cady et al. 1993; Overgaard Nielsen et al. 1999). There are very few records of acrocerids parasitizing true web-dwelling spiders and exceptions typically involve comb-footed spiders (e.g., Theridiidae), where the spider may still be proximal to the substrate (e.g., Lamore 1960).
2.4.2 Family Sarcophagidae: As discussed above, the majority of members of Sarcophagidae are generalist scavengers and insect predators (Pape et al. 2012). However, there is one example of a sarcophagid acting as an endoparasitoid of a scorpion. Sarcophaga dux (Thomson, 1869), a species generally considered to be of forensic importance, was found to attack the Chinese scorpion Mesobuthus martensii (Karsch, 1879) (Scorpiones: Buthidae). Multiple larvae (3-6) attacked more than 50 individuals of this scorpion species, with more than 100 flies emerging as adults by the end of the study (Shi et al. 2015).

2.4.3 Family Tachinidae: Species of Tachinidae are internal parasitoids on a wide range of arthropod hosts. The most commonly used hosts are phytophagous insects, primarily Lepidoptera, Coleoptera, Hymenoptera, Heteroptera, and Orthoptera. Several genera of tachinids attack non-insect arthropods, including centipedes and scorpions (Stireman et al. 2006). There is a single record of a spider serving as a tachinid host (Vincent 1985). Two tachinid larvae in the genus Lypha Robineau-Desvoidy, 1830 emerged from the abdomen of immature individuals of Antrodiaetus riversi (O. PickardCambridge, 1883) in the laboratory. The two larvae pupated outside of the host but neither adult emerged. Other known hosts of Lypha species are Lepidoptera in the families Gelechiidae and Tortricidae, and it is likely that the two spiders may have been accidental hosts that did not manage to survive and become adults. Vincent (1985) reported rearing over 340 spiders, which were examined and inspected for signs of parasitism, and only two contained larval tachinids.

The tachinid Spilochaetosoma californicum Smith, 1917 has been found to be an endoparasitoid of two Nearctic scorpions, Anuroctonus phaiodactylus (Wood, 1863) and Paravaejovis spinigerus (Wood, 1863) (Scorpiones: Vaejovidae). Multiple larvae of $S$. californicum emerged from wild caught individuals of both species and it is likely that this tachinid is a general parasitoid of burrowing scorpions (Williams et al. 1994).

\section{CONCLUSION}

In this review we summarized the available information on all lineages of Diptera known to attack arachnids, including predators, parasites, kleptoparasites and parasitoids. A summary table (Table 1) containing over 200 host records for eight families of Diptera attacking 36 arachnid families is included. Even though hymenopterans are among the best known natural enemies of arachnids, species of Diptera clearly also comprise a large component of arachnid enemies, attacking families in four orders of arachnids and all three suborders of Araneae.

A single species of Sarcophagidae, one species of Tachinidae and all species of Acroceridae are internal parasitoids, attacking juvenile and/or adult arachnids. Spider flies (family Acroceridae) comprise the only lineage of Diptera known to develop exclusively on arachnid hosts, representing some of the most significant natural enemies of spiders. Multiple species of Chloropidae, Drosophilidae, Ephydridae, Phoridae and Sarcophagidae are known to attack eggs of 11 families of arachnids, acting as predators and/or parasitoids of arachnid egg sacs (see Table I). Some of these species are known to be true predators, while some are known to be true parasitoids. However, in most cases, the life history strategy is not clear, 
Table 1.- Host records for the known natural enemies of arachnids in the order Diptera, organized by arachnid order and family. Due to their generalist feeding habits, predators and kleptoparasites of individual arachnids were not included. Host stage exploited by the dipteran is noted as egg, adult, immature or stage unknown (Adult/Immature). EIS = E.I. Schlinger Collection database record.

\begin{tabular}{|c|c|c|c|c|c|c|}
\hline Host order & Host family & Arachnid host & Host stage & Fly family & $\begin{array}{l}\text { Fly parasitoid/ } \\
\text { parasite/predator }\end{array}$ & Reference \\
\hline \multirow[t]{2}{*}{ Amblypygi } & \multirow[t]{2}{*}{ Phrynidae } & $\begin{array}{l}\text { Paraphrynus laevifrons } \\
\text { (Pocock, 1894) }\end{array}$ & Adult & Chloropidae & Pseudogaurax sp. & $\begin{array}{l}\text { Viquez \& De Armas } \\
2009\end{array}$ \\
\hline & & $\begin{array}{l}\text { Phrynus } \\
\text { pseudoparvulus } \\
\text { Armas \& Viquez, } \\
2001\end{array}$ & Adult & Chloropidae & Pseudogaurax sp. & $\begin{array}{l}\text { Viquez \& De Armas } \\
\quad 2009\end{array}$ \\
\hline \multirow[t]{18}{*}{ Araneae } & \multirow[t]{18}{*}{ Agelenidae } & $\begin{array}{l}\text { Agelenopsis naevia } \\
\quad \text { (Walckenaer, 1841) }\end{array}$ & $\begin{array}{l}\text { Adult/ } \\
\text { Immature }\end{array}$ & Acroceridae & $\begin{array}{l}\text { Turbopsebius } \\
\quad \text { sulphuripes (Loew) }\end{array}$ & Melander 1902 \\
\hline & & $\begin{array}{l}\text { Agelenopsis } \\
\text { oregonensis } \\
\text { Chamberlin \& Ivie, } \\
1935\end{array}$ & $\begin{array}{l}\text { Adult/ } \\
\text { Immature }\end{array}$ & Acroceridae & $\begin{array}{l}\text { Acrocera bakeri } \\
\text { Coquillett }\end{array}$ & Schlinger 1987 \\
\hline & & Agelenopsis sp. & $\begin{array}{l}\text { Adult/ } \\
\text { Immature }\end{array}$ & Acroceridae & $\begin{array}{l}\text { Acrocera melanderi } \\
\text { Cole }\end{array}$ & EIS database \\
\hline & & Agelenopsis sp. & $\begin{array}{l}\text { Adult/ } \\
\text { Immature }\end{array}$ & Acroceridae & $\begin{array}{l}\text { Ogcodes dispar } \\
\text { (Macquart) }\end{array}$ & Schlinger 1987 \\
\hline & & Agelenopsis sp. & Immature & Acroceridae & $\begin{array}{l}\text { Ogcodes eugonatus } \\
\text { (Loew) }\end{array}$ & Guarisco 1990 \\
\hline & & Barronopsis sp. & $\begin{array}{l}\text { Adult/ } \\
\text { Immature }\end{array}$ & Acroceridae & $\begin{array}{l}\text { Acrocera bimaculata } \\
\text { (Loew) }\end{array}$ & Schlinger 1987 \\
\hline & & $\begin{array}{l}\text { Coras montanus } \\
\quad(\text { Emerton, 1890) }\end{array}$ & $\begin{array}{l}\text { Adult/ } \\
\text { Immature }\end{array}$ & Acroceridae & $\begin{array}{l}\text { Acrocera bimaculata } \\
\text { (Loew) }\end{array}$ & Cady et al. 1993 \\
\hline & & Coras montanus & $\begin{array}{l}\text { Adult/ } \\
\text { Immature }\end{array}$ & Acroceridae & $\begin{array}{l}\text { Pterodontia flavipes } \\
\text { Gray }\end{array}$ & Sabrosky 1948 \\
\hline & & Coras montanus & $\begin{array}{l}\text { Adult/ } \\
\text { Immature }\end{array}$ & Acroceridae & $\begin{array}{l}\text { Turbopsebius } \\
\quad \text { sulphuripes (Loew) }\end{array}$ & Cady et al. 1993 \\
\hline & & $\begin{array}{l}\text { Eratigena sicana } \\
\text { (Brignoli,1976) }\end{array}$ & $\begin{array}{l}\text { Adult/ } \\
\text { Immature }\end{array}$ & Acroceridae & Ogcodes sp. & Brignoli 1976 \\
\hline & & $\begin{array}{l}\text { Hololena curta } \\
\qquad(\text { McCook, 1894) }\end{array}$ & $\begin{array}{l}\text { Aduit/ } \\
\text { Immature }\end{array}$ & Acroceridae & $\begin{array}{l}\text { Acrocera subfasciata } \\
\text { Westwood }\end{array}$ & EIS database \\
\hline & & Hololena curta & Immature & Acroceridae & $\begin{array}{l}\text { Ogcodes adaptatus } \\
\text { Schlinger }\end{array}$ & Schlinger 1960 \\
\hline & & Hololena curta & $\begin{array}{l}\text { Adult and } \\
\text { immature }\end{array}$ & Acroceridae & $\begin{array}{l}\text { Ogcodes pallidipennis } \\
\text { (Loew) }\end{array}$ & Schlinger 1960 \\
\hline & & Hololena curta & Immature & Acroceridae & $\begin{array}{l}\text { Turbopsebius diligens } \\
\text { (Osten Sacken) }\end{array}$ & Schlinger 1952 \\
\hline & & Hololena sp. & $\begin{array}{l}\text { Adult/ } \\
\text { Immature }\end{array}$ & Acroceridae & $\begin{array}{l}\text { Acrocera melanderi } \\
\text { Cole }\end{array}$ & Cole 1969 \\
\hline & & Hololena sp. & $\begin{array}{l}\text { Adult/ } \\
\text { Immature }\end{array}$ & Acroceridae & $\begin{array}{l}\text { Acrocera subfasciata } \\
\text { Westwood }\end{array}$ & Schlinger 1987 \\
\hline & & Rualena sp. & $\begin{array}{l}\text { Adult/ } \\
\text { Immature }\end{array}$ & Acroceridae & $\begin{array}{l}\text { Turbopsebius diligens } \\
\text { (Osten Sacken) }\end{array}$ & Schlinger 1987 \\
\hline & & $\begin{array}{l}\text { Textrix denticulata } \\
\text { (Olivier, 1769) }\end{array}$ & $\begin{array}{l}\text { Adult/ } \\
\text { Immature }\end{array}$ & Acroceridae & $\begin{array}{l}\text { Acrocera sanguinea } \\
\text { Meigen }\end{array}$ & Koch 1872 \\
\hline \multirow[t]{5}{*}{ Araneae } & \multirow[t]{5}{*}{ Amaurobiidae } & $\begin{array}{l}\text { Amaurobius erberi } \\
\text { (Keyserling, 1893) }\end{array}$ & Immature & Acroceridae & $\begin{array}{l}\text { Acrocera orbicula } \\
\text { (Fabricius) }\end{array}$ & $\begin{array}{l}\text { Kehlmaier \& } \\
\text { Almeida } 2014\end{array}$ \\
\hline & & Amaurobius sp. & Immature & Acroceridae & $\begin{array}{l}\text { Ogcodes pallidipennis } \\
\text { (Loew) }\end{array}$ & Schlinger 1960 \\
\hline & & $\begin{array}{l}\text { Callobius bennetti } \\
\quad \text { (Blackwall, 1846) }\end{array}$ & $\begin{array}{l}\text { Adult/ } \\
\text { Immature }\end{array}$ & Acroceridae & $\begin{array}{l}\text { Acrocera fasciata } \\
\text { Wiedemann }\end{array}$ & Emerton 1890 \\
\hline & & Undetermined genus & $\begin{array}{l}\text { Adult/ } \\
\text { Immature }\end{array}$ & Acroceridae & $\begin{array}{l}\text { Megalybus pictus } \\
\text { Philippi }\end{array}$ & Schlinger 1987 \\
\hline & & Undetermined genus & $\begin{array}{l}\text { Adult/ } \\
\text { Immature }\end{array}$ & Acroceridae & Thyllis $\mathrm{sp}$. & Schlinger 2003 \\
\hline Araneae & Amphinectidae & Metaltella sp. & $\begin{array}{l}\text { Adult/ } \\
\text { Immature }\end{array}$ & Acroceridae & $\begin{array}{l}\text { Holops cyaneus } \\
\text { Philippi }\end{array}$ & Schlinger 1987 \\
\hline \multirow[t]{2}{*}{ Araneae } & \multirow[t]{2}{*}{ Antrodiaetidae } & $\begin{array}{l}\text { Aliatypus californicus } \\
\text { (Banks, 1896) }\end{array}$ & $\begin{array}{l}\text { Adult/ } \\
\text { Immature }\end{array}$ & Acroceridae & Eulonchus tristis Loew & $\begin{array}{l}\text { Coyle \& Icenogle } \\
1994\end{array}$ \\
\hline & & $\begin{array}{l}\text { Aliatypus erebus } \\
\text { Coyle, } 1974\end{array}$ & Adult & Acroceridae & Eulonchus tristis Loew & $\begin{array}{l}\text { Coyle \& Icenogle } \\
1994\end{array}$ \\
\hline
\end{tabular}


Table 1.-Continued.

\begin{tabular}{|c|c|c|c|c|c|c|}
\hline Host order & Host family & Arachnid host & Host stage & Fly family & $\begin{array}{l}\text { Fly parasitoid/ } \\
\text { parasite/predator }\end{array}$ & Reference \\
\hline \multirow[t]{6}{*}{ Araneae } & \multirow[t]{6}{*}{ Antrodiaetidae } & Aliatypus sp. & $\begin{array}{l}\text { Adult/ } \\
\text { Immature }\end{array}$ & Acroceridae & Eulonchus tristis Loew & Schlinger 1987 \\
\hline & & $\begin{array}{l}\text { Antrodiaetus riversi } \\
\text { (O. Pickard- } \\
\text { Cambridge, 1883) }\end{array}$ & $\begin{array}{l}\text { Adult/ } \\
\text { Immature }\end{array}$ & Acroceridae & $\begin{array}{l}\text { Eulonchus sapphirinus } \\
\text { Osten Sacken }\end{array}$ & EIS database \\
\hline & & Antrodiaetus riversi & $\begin{array}{l}\text { Adult/ } \\
\text { Immature }\end{array}$ & Acroceridae & Eulonchus sp. & Vincent 1986 \\
\hline & & Antrodiaetus riversi & Adult & Tachinidae & Lypha sp. & Vincent 1985 \\
\hline & & $\begin{array}{l}\text { Antrodiaetus unicolor } \\
\text { (Hentz, 1842) }\end{array}$ & $\begin{array}{l}\text { Adult/ } \\
\text { Immature }\end{array}$ & Acroceridae & $\begin{array}{l}\text { Eulonchus marialiciae } \\
\text { Brimley }\end{array}$ & $\begin{array}{l}\text { Coyle 1971; Adler et } \\
\text { al } 1997\end{array}$ \\
\hline & & Antrodiaetus sp. & $\begin{array}{l}\text { Adult/ } \\
\text { Immature }\end{array}$ & Acroceridae & Eulonchus sp. & Schlinger 1987 \\
\hline \multirow[t]{3}{*}{ Araneae } & \multirow[t]{3}{*}{ Anyphaenidae } & $\begin{array}{l}\text { Anyphaena californica } \\
\text { (Banks, 1904) }\end{array}$ & $\begin{array}{l}\text { Adult/ } \\
\text { Immature }\end{array}$ & Acroceridae & Ogcodes sp. & Cady et al. 1993 \\
\hline & & $\begin{array}{l}\text { Wulfila saltabundus } \\
\text { (Hentz, 1847) }\end{array}$ & $\begin{array}{l}\text { Adult/ } \\
\text { Immature }\end{array}$ & Acroceridae & Ogcodes borealis Cole & Sabrosky 1948 \\
\hline & & Wulfila saltabundus & $\begin{array}{l}\text { Adult/ } \\
\text { Immature }\end{array}$ & Acroceridae & $\begin{array}{l}\text { Ogcodes pallidipennis } \\
\text { (Loew) }\end{array}$ & Sabrosky 1948 \\
\hline \multirow[t]{18}{*}{ Araneae } & \multirow[t]{18}{*}{ Araneidae } & $\begin{array}{l}\text { Alpaida veniliae } \\
\text { (Keyserling,1865) }\end{array}$ & Egg & Chloropidae & $\begin{array}{l}\text { Pseudogaurax } \\
\text { cingulatus Sabrosky }\end{array}$ & Sabrosky 1966 \\
\hline & & $\begin{array}{l}\text { Araneus angulatus } \\
\text { Clerck, } 1757\end{array}$ & Egg & Chloropidae & $\begin{array}{l}\text { Pseudogaurax signatus } \\
\text { (Loew) }\end{array}$ & Davidson 1896 \\
\hline & & $\begin{array}{c}\text { Araneus ejusmodi } \\
\text { (Boesenberg \& } \\
\text { Strand, 1906) }\end{array}$ & Egg & Chloropidae & $\begin{array}{l}\text { Gaurax chiyokae } \\
\text { (Kanmiya) }\end{array}$ & Kanmiya 1972, 1983 \\
\hline & & $\begin{array}{l}\text { Araneus gemma } \\
\quad \text { (McCook, 1888) }\end{array}$ & Egg & Chloropidae & $\begin{array}{l}\text { Pseudogaurax signatus } \\
\text { (Loew) }\end{array}$ & Pierce 1942 \\
\hline & & $\begin{array}{l}\text { Argiope aemula } \\
\quad \text { (Walckenaer, 1841) }\end{array}$ & Egg & Phoridae & $\begin{array}{l}\text { Megaselia araneivora } \\
\text { Goto }\end{array}$ & Goto 1985 \\
\hline & & $\begin{array}{l}\text { Argiope argentata } \\
\text { (Fabricius, } 1775 \text { ) }\end{array}$ & Egg & Sarcophagidae & $\begin{array}{l}\text { Sarcophaga davidsonii } \\
\text { Coquillett }\end{array}$ & Davidson 1894 \\
\hline & & $\begin{array}{l}\text { Argiope aurantia } \\
\text { Lucas, } 1833\end{array}$ & Egg & Phoridae & Megaselia sp. & $\begin{array}{l}\text { Kaston \& Jenks } \\
1937\end{array}$ \\
\hline & & Argiope aurantia & Egg & Chloropidae & $\begin{array}{l}\text { Pseudogaurax anchora } \\
\text { (Loew) }\end{array}$ & $\begin{array}{l}\text { Kaston \& Jenks } \\
1937\end{array}$ \\
\hline & & Argiope aurantia & Egg & Chloropidae & $\begin{array}{l}\text { Pseudogaurax signatus } \\
\text { (Loew) }\end{array}$ & Coquillet 1898 \\
\hline & & Argiope aurantia & Egg & Chloropidae & $\begin{array}{l}\text { Pseudogaurax signatus } \\
\text { (Loew) }\end{array}$ & $\begin{array}{l}\text { Lockley \& Young } \\
1993\end{array}$ \\
\hline & & Argiope aurantia & Egg & Sarcophagidae & $\begin{array}{l}\text { Sarcophaga litsingeri } \\
\text { (Shinonaga \& } \\
\text { Barrion) }\end{array}$ & Davidson 1896 \\
\hline & & $\begin{array}{l}\text { Argiope catenulata } \\
\text { (Doleschall, 1859) }\end{array}$ & Egg & Sarcophagidae & $\begin{array}{l}\text { Sarcophaga litsingeri } \\
\text { (Shinonaga \& } \\
\text { Barrion) }\end{array}$ & $\begin{array}{l}\text { Shinonaga \& } \\
\text { Barrion } 1980\end{array}$ \\
\hline & & $\begin{array}{l}\text { Argiope pulchella } \\
\text { Thorell, } 1881\end{array}$ & Egg & Sarcophagidae & $\begin{array}{l}\text { Sarcophaga banksi } \\
\text { Senior-White }\end{array}$ & $\begin{array}{l}\text { Prakash \& Pandian } \\
1977\end{array}$ \\
\hline & & $\begin{array}{l}\text { Argiope trifasciata } \\
\text { (Forsskål, 1775) }\end{array}$ & Egg & Sarcophagidae & $\begin{array}{l}\text { Tricharaea } \\
\text { (Sarcophagula) } \\
\text { Thomson }\end{array}$ & $\begin{array}{l}\text { de Armas \& Garcia } \\
1986\end{array}$ \\
\hline & & Argiope sp. & Egg & Phoridae & $\begin{array}{l}\text { Megaselia } \\
\text { argiopephaga } \\
\text { Disney }\end{array}$ & Disney 1982 \\
\hline & & $\begin{array}{l}\text { Cyrtophora } \\
\text { moluccensis } \\
\text { (Doleschall, 1857) }\end{array}$ & Egg & Sarcophagidae & $\begin{array}{l}\text { Sarcophaga } \\
\quad \text { arachnivora (Lopes) }\end{array}$ & Cantrell 1986 \\
\hline & & $\begin{array}{l}\text { Cyrtophora } \\
\text { moluccensis }\end{array}$ & Egg & Sarcophagidae & $\begin{array}{l}\text { Sarcophaga } \\
\text { cyrtophorae } \\
\text { (Cantrell) }\end{array}$ & Cantrell 1986 \\
\hline & & $\begin{array}{l}\text { Cyrtophora } \\
\text { moluccensis }\end{array}$ & Egg & Sarcophagidae & $\begin{array}{l}\text { Sarcophaga reposita } \\
\text { (Lopes) }\end{array}$ & Cantrell 1986 \\
\hline
\end{tabular}


Table 1.-Continued.

\begin{tabular}{|c|c|c|c|c|c|c|}
\hline Host order & Host family & Arachnid host & Host stage & Fly family & $\begin{array}{l}\text { Fly parasitoid/ } \\
\text { parasite/predator }\end{array}$ & Reference \\
\hline \multirow[t]{18}{*}{ Araneae } & \multirow[t]{18}{*}{ Araneidae } & $\begin{array}{l}\text { Gasteracantha } \\
\quad \text { cancriformis } \\
\text { (Linnaeus, 1785) }\end{array}$ & Egg & Phoridae & $\begin{array}{l}\text { Phalacrotophora } \\
\text { epeirae (Brues) }\end{array}$ & Muma \& Stone 1971 \\
\hline & & $\begin{array}{l}\text { Gasteracantha } \\
\text { cancriformis }\end{array}$ & Egg & Chloropidae & $\begin{array}{l}\text { Pseudogaurax lancifer } \\
\text { (Coquillett) }\end{array}$ & Hall 1937 \\
\hline & & $\begin{array}{l}\text { Larinioides cornutus } \\
\text { (Clerck, 1757) }\end{array}$ & Egg & Chloropidae & $\begin{array}{l}\text { Conioscinella frontella } \\
\text { (Fallen) }\end{array}$ & Krijger 1910 \\
\hline & & Larinioides cornutus & Egg & Chloropidae & $\begin{array}{l}\text { Oscinella halterata } \\
\text { (Lamb) }\end{array}$ & Auten 1925 \\
\hline & & Larinioides cornutus & Egg & Sarcophagidae & $\begin{array}{l}\text { Sarcophaga } \\
\text { sexpunctata } \\
\text { (Fabricius) }\end{array}$ & Auten 1925 \\
\hline & & Larinioides cornutus & Egg & Tachinidae & Tachina sp. & Bertkau 1880 \\
\hline & & $\begin{array}{l}\text { Larinioides } \\
\text { sclopetarius (Clerck, } \\
\text { 1757) }\end{array}$ & Egg & Chloropidae & $\begin{array}{l}\text { Oscinella halterata } \\
\text { (Lamb) }\end{array}$ & Auten 1925 \\
\hline & & $\begin{array}{l}\text { Larinioides } \\
\text { sclopetarius }\end{array}$ & Egg & Phoridae & $\begin{array}{l}\text { Phalacrotophora } \\
\text { epeirae (Brues) }\end{array}$ & $\begin{array}{l}\text { Brues 1902, 1903; } \\
\text { Auten } 1925\end{array}$ \\
\hline & & $\begin{array}{l}\text { Larinioides } \\
\text { sclopetarius }\end{array}$ & $\begin{array}{l}\text { Adult/ } \\
\text { Immature }\end{array}$ & Acroceridae & $\begin{array}{l}\text { Pterodontia flavipes } \\
\text { Gray }\end{array}$ & King 1916 \\
\hline & & $\begin{array}{l}\text { Larinioides } \\
\quad \text { sclopetarius }\end{array}$ & Egg & Sarcophagidae & $\begin{array}{l}\text { Sarcophaga } \\
\text { sexpunctata } \\
\text { (Fabricius) }\end{array}$ & Auten 1925 \\
\hline & & $\begin{array}{c}\text { Mecynogea lemniscata } \\
\quad \text { (Walckenaer, 1841) }\end{array}$ & Adult & Acroceridae & $\begin{array}{l}\text { Ogcodes dispar } \\
\text { Macquart }\end{array}$ & Lamore 1960 \\
\hline & & $\begin{array}{l}\text { Metepeira atascadero } \\
\text { Piel, } 2001\end{array}$ & Egg & Sarcophagidae & $\begin{array}{l}\text { Sarcophaga lindae } \\
\text { (Lopes) }\end{array}$ & Hieber \& Uetz 1990 \\
\hline & & $\begin{array}{l}\text { Metepeira incrassata } \\
\text { O. Pickard- } \\
\text { Cambridge, } 1903\end{array}$ & Egg & Sarcophagidae & $\begin{array}{l}\text { Sarcophaga lindae } \\
\text { (Lopes) }\end{array}$ & Hieber \& Uetz 1990 \\
\hline & & $\begin{array}{l}\text { Neoscona nautica }(\mathrm{L} . \\
\text { Koch, 1875) }\end{array}$ & Egg & Chloropidae & $\begin{array}{c}\text { Gaurax chiyokae } \\
\text { (Kanmiya) }\end{array}$ & Kanmiya 1972, 1983 \\
\hline & & $\begin{array}{l}\text { Ordgarius magnificus } \\
\quad \text { (Rainbow, 1897) }\end{array}$ & Egg & Sarcophagidae & $\begin{array}{l}\text { Sarcophaga } \\
\quad \text { arachnivora (Lopes) }\end{array}$ & Souza Lopes 1985 \\
\hline & & $\begin{array}{l}\text { Singa nitidula C. L. } \\
\text { Koch, } 1844\end{array}$ & Egg & Chloropidae & $\begin{array}{l}\text { Conioscinella frontella } \\
\text { (Fallen) }\end{array}$ & Vachon 1952 \\
\hline & & $\begin{array}{l}\text { Zygiella } x \text {-notata } \\
\text { (Clerck, 1757) }\end{array}$ & $\begin{array}{l}\text { Adult/ } \\
\text { Immature }\end{array}$ & Acroceridae & $\begin{array}{l}\text { Ogcodes fumatus } \\
\text { (Erichson) }\end{array}$ & Holl et al. 1983 \\
\hline & & Undetermined genus & Egg & Phoridae & $\begin{array}{l}\text { Megaselia longifurca } \\
\text { (Lundbeck) }\end{array}$ & Disney 1999 \\
\hline \multirow[t]{4}{*}{ Araneae } & \multirow[t]{4}{*}{ Clubionidae } & $\begin{array}{l}\text { Clubiona leucaspis } \\
\text { (Simon, 1932) }\end{array}$ & Immature & Acroceridae & $\begin{array}{l}\text { Ogcodes reginae } \\
\text { Trojan }\end{array}$ & $\begin{array}{l}\text { Kehlmaier \& } \\
\text { Almeida } 2014\end{array}$ \\
\hline & & $\begin{array}{l}\text { Clubiona putris nom. } \\
\text { dub. C. L. Koch, } \\
1839\end{array}$ & $\begin{array}{l}\text { Adult/ } \\
\text { Immature }\end{array}$ & Acroceridae & $\begin{array}{l}\text { Ogcodes eugonatus } \\
\text { (Loew) }\end{array}$ & Emerton 1890 \\
\hline & & $\begin{array}{l}\text { Clubiona putris nom. } \\
\text { dub. }\end{array}$ & $\begin{array}{l}\text { Aduit/ } \\
\text { Immature }\end{array}$ & Acroceridae & $\begin{array}{l}\text { Ogcodes pallipes } \\
\text { Latreille }\end{array}$ & Menge 1866 \\
\hline & & Clubiona sp. & Immature & Acroceridae & $\begin{array}{l}\text { Acrocera orbicula } \\
\text { (Fabricius) }\end{array}$ & Millot 1938 \\
\hline \multirow[t]{2}{*}{ Araneae } & \multirow[t]{2}{*}{ Ctenizidae } & $\begin{array}{l}\text { Bothriocyrtum } \\
\text { californicum (O. } \\
\text { Pickard-Cambridge, } \\
\text { 1874) }\end{array}$ & $\begin{array}{l}\text { Adult/ } \\
\text { Immature }\end{array}$ & Acroceridae & $\begin{array}{l}\text { Ocnaea smithi } \\
\text { Sabrosky }\end{array}$ & Jenks 1938,1940 \\
\hline & & $\begin{array}{l}\text { Cyrtocarenum } \\
\text { cunicularium } \\
\text { (Olivier, 1811) }\end{array}$ & $\begin{array}{l}\text { Adult/ } \\
\text { Immature }\end{array}$ & Acroceridae & $\begin{array}{l}\text { Astomella hispaniae } \\
\text { Lamarck }\end{array}$ & Brauer 1869 \\
\hline Araneae & Desidae & $\begin{array}{l}\text { Matachia ramulicola } \\
\text { Dalmas, } 1917\end{array}$ & $\begin{array}{l}\text { Adult/ } \\
\text { Immature }\end{array}$ & Acroceridae & $\begin{array}{l}\text { Ogcodes brunneus } \\
\text { (Hutton) }\end{array}$ & Dumbleton 1940 \\
\hline Araneae & Dipluridae & $\begin{array}{l}\text { Linothele cousini } \\
\text { (Simon, 1889) }\end{array}$ & $\begin{array}{l}\text { Adult/ } \\
\text { Immature }\end{array}$ & Acroceridae & $\begin{array}{l}\text { Lasia ecuadorensis } \\
\text { Bequaert }\end{array}$ & Schlinger 1987 \\
\hline Araneae & Euctenizidae & $\begin{array}{l}\text { Aptostichus } \\
\text { stanfordianus Smith, } \\
1908\end{array}$ & $\begin{array}{l}\text { Adult/ } \\
\text { Immature }\end{array}$ & Acroceridae & $\begin{array}{l}\text { Eulonchus smaragdinus } \\
\text { Gerstaecker }\end{array}$ & Schlinger 1987 \\
\hline
\end{tabular}


Table 1.-Continued.

\begin{tabular}{|c|c|c|c|c|c|c|}
\hline Host order & Host family & Arachnid host & Host stage & Fly family & $\begin{array}{l}\text { Fly parasitoid/ } \\
\text { parasite/predator }\end{array}$ & Reference \\
\hline \multirow[t]{3}{*}{ Araneae } & \multirow[t]{3}{*}{ Eutichuridae } & $\begin{array}{l}\text { Cheiracanthium } \\
\text { punctorium (Villers, } \\
1789 \text { ) }\end{array}$ & Egg & Sarcophagidae & $\begin{array}{l}\text { Sarcophaga } \\
\text { sexpunciata } \\
\text { (Fabricius) }\end{array}$ & $\begin{array}{l}\text { Krehenwinkel et al. } \\
2016\end{array}$ \\
\hline & & Cheiracanthium sp. & $\begin{array}{l}\text { Adult/ } \\
\text { Immature }\end{array}$ & Acroceridae & $\begin{array}{l}\text { Ogcodes croucampi } \\
\text { Barraclough }\end{array}$ & $\begin{array}{l}\text { Barraclough \& } \\
\text { Croucamp } 1997\end{array}$ \\
\hline & & Cheiracanthium sp. & Immature & Acroceridae & Ogcodes sp. & Schlinger 2003 \\
\hline \multirow[t]{3}{*}{ Araneae } & \multirow[t]{3}{*}{ Gnaphosidae } & Herpyllus sp. & Immature & Acroceridae & $\begin{array}{l}\text { Ogcodes pallidipennis } \\
\text { (Loew) }\end{array}$ & Schlinger 1960 \\
\hline & & Zelotes sp. & $\begin{array}{l}\text { Adult/ } \\
\text { Immature }\end{array}$ & Acroceridae & $\begin{array}{l}\text { Acrocera melanderi } \\
\text { Cole }\end{array}$ & Schlinger 1987 \\
\hline & & Zelotes sp. & $\begin{array}{l}\text { Adult/ } \\
\text { Immature }\end{array}$ & Acroceridae & $\begin{array}{l}\text { Ogcodes gibbosus } \\
\text { (Linnaeus) }\end{array}$ & Nielsen 1932 \\
\hline \multirow[t]{2}{*}{ Araneae } & \multirow[t]{2}{*}{ Linyphiidae } & $\begin{array}{l}\text { Hypselistes florens (O. } \\
\text { Pickard-Cambridge, } \\
\text { 1875) }\end{array}$ & Egg & Ephydridae & $\begin{array}{l}\text { Trimerina madizans } \\
\text { (Fallen) }\end{array}$ & Wirth et al. 1987 \\
\hline & & $\begin{array}{l}\text { Pityohyphantes } \\
\text { costatus (Hentz, } \\
1850 \text { ) }\end{array}$ & Egg & Phoridae & $\begin{array}{l}\text { Phalacrotophora } \\
\text { epeirae (Brues) }\end{array}$ & Manuel 1984 \\
\hline \multirow[t]{2}{*}{ Araneae } & \multirow[t]{2}{*}{ Liphistiidae } & $\begin{array}{l}\text { Liphistius lahu } \\
\quad \text { Schwendinger, } 1998\end{array}$ & $\begin{array}{l}\text { Adult/ } \\
\text { Immature }\end{array}$ & Sarcophagidae & Metopia sinensis Pape & $\begin{array}{l}\text { Schwendinger \& } \\
\text { Pape } 2000\end{array}$ \\
\hline & & Liphistius sp. & Adult & Sarcophagidae & Metopia sinensis Pape & $\begin{array}{l}\text { Schwendinger \& } \\
\text { Pape } 2000\end{array}$ \\
\hline \multirow[t]{21}{*}{ Araneae } & \multirow[t]{21}{*}{ Lycosidae } & $\begin{array}{l}\text { Alopecosa accentuata } \\
\text { (Latreille, 1817) }\end{array}$ & $\begin{array}{l}\text { Adult/ } \\
\text { Immature }\end{array}$ & Acroceridae & $\begin{array}{l}\text { Ogcodes pallipes } \\
\text { Latreille }\end{array}$ & Eason et al. 1967 \\
\hline & & $\begin{array}{l}\text { Alopecosa barbipes } \\
\text { (Sundevall, 1833) }\end{array}$ & Adult & Acroceridae & $\begin{array}{l}\text { Ogcodes gibbosus } \\
\text { (Linnaeus) }\end{array}$ & Locket 1930 \\
\hline & & Alopecosa barbipes & $\begin{array}{l}\text { Adult/ } \\
\text { Immature }\end{array}$ & Acroceridae & $\begin{array}{l}\text { Ogcodes pallipes } \\
\text { Latreille }\end{array}$ & Locket 1930 \\
\hline & & $\begin{array}{l}\text { Alopecosa kochi } \\
\text { (Keyserling, 1877) }\end{array}$ & Immature & Acroceridae & $\begin{array}{l}\text { Ogcodes melampus } \\
\text { (Loew) }\end{array}$ & Schlinger 1960 \\
\hline & & $\begin{array}{l}\text { Geolycosa domifex } \\
\text { (Hancock, 1899) }\end{array}$ & $\begin{array}{l}\text { Adult/ } \\
\text { Immature }\end{array}$ & Acroceridae & $\begin{array}{l}\text { Pterodontia flavipes } \\
\text { Gray }\end{array}$ & McQueen 1978, 1983 \\
\hline & & $\begin{array}{l}\text { Lycosa godeffroyi } \mathrm{L} \text {. } \\
\text { Koch, } 1865\end{array}$ & Immature & Acroceridae & $\begin{array}{l}\text { Ogcodes basalis } \\
\text { (Walker) }\end{array}$ & Humphreys 1976 \\
\hline & & Lycosa godeffroyi & Immature & Acroceridae & $\begin{array}{l}\text { Pterodontia melli } \\
\text { Erichson }\end{array}$ & Humphreys 1976 \\
\hline & & $\begin{array}{l}\text { Pardosa alacris (C. L. } \\
\text { Koch, 1833) }\end{array}$ & Adult & Acroceridae & $\begin{array}{l}\text { Ogcodes gibbosus } \\
\text { (Linnaeus) }\end{array}$ & Langer 2005 \\
\hline & & $\begin{array}{l}\text { Pardosa distincta } \\
\quad \text { (Blackwall, 1846) }\end{array}$ & $\begin{array}{l}\text { Adult/ } \\
\text { Immature }\end{array}$ & Acroceridae & $\begin{array}{l}\text { Ogcodes eugonatus } \\
\text { (Loew) }\end{array}$ & Sabrosky 1948 \\
\hline & & $\begin{array}{c}\text { Pardosa lapidicina } \\
\text { Emerton, } 1885\end{array}$ & $\begin{array}{l}\text { Adult/ } \\
\text { Immature }\end{array}$ & Acroceridae & $\begin{array}{l}\text { Acrocera fasciata } \\
\text { Wiedemann }\end{array}$ & Eason 1966 \\
\hline & & Pardosa lapidicina & $\begin{array}{l}\text { Adult/ } \\
\text { Immature }\end{array}$ & Acroceridae & $\begin{array}{l}\text { Ogcodes pallidipennis } \\
\text { (Loew) }\end{array}$ & Eason 1966 \\
\hline & & $\begin{array}{l}\text { Pardosa littoralis } \\
\text { Banks, } 1846\end{array}$ & Immature & Acroceridae & $\begin{array}{l}\text { Ogcodes eugonatus } \\
\text { (Loew) }\end{array}$ & Kaston 1937 \\
\hline & & Pardosa littoralis & Immature & Acroceridae & $\begin{array}{l}\text { Ogcodes pallidipennis } \\
\text { (Loew) }\end{array}$ & Kaston 1937 \\
\hline & & $\begin{array}{l}\text { Pardosa lugubris } \\
\text { (Walckenaer, 1802) }\end{array}$ & $\begin{array}{l}\text { Adult/ } \\
\text { Immature }\end{array}$ & Acroceridae & $\begin{array}{l}\text { Ogcodes fumatus } \\
\text { (Erichson) }\end{array}$ & de Jong et al. 2000 \\
\hline & & $\begin{array}{r}\text { Pardosa milvina } \\
\text { (Hentz, 1844) }\end{array}$ & $\begin{array}{l}\text { Adult/ } \\
\text { Immature }\end{array}$ & Acroceridae & $\begin{array}{l}\text { Ogcodes eugonatus } \\
\text { (Loew) }\end{array}$ & Eason et al. 1967 \\
\hline & & Pardosa milvina & Immature & Acroceridae & Undetermined genus & $\begin{array}{l}\text { Allard \& Robertson } \\
2003\end{array}$ \\
\hline & & $\begin{array}{l}\text { Pardosa prativaga (L. } \\
\text { Koch, 1870) }\end{array}$ & Immature & Acroceridae & $\begin{array}{l}\text { Acrocera orbicula } \\
\text { (Fabricius) }\end{array}$ & Toft et al. 2012 \\
\hline & & $\begin{array}{l}\text { Pardosa pullata } \\
\text { (Clerck, 1757) }\end{array}$ & $\begin{array}{l}\text { Adult/ } \\
\text { Immature }\end{array}$ & Acroceridae & $\begin{array}{l}\text { Ogcodes gibbosus } \\
\text { (Linnaeus) }\end{array}$ & Duffey 2000 \\
\hline & & Pardosa pullata & $\begin{array}{l}\text { Adult/ } \\
\text { Immature }\end{array}$ & Acroceridae & $\begin{array}{l}\text { Ogcodes pallipes } \\
\text { Latreille }\end{array}$ & Schlinger 1987 \\
\hline & & Pardosa pullata & $\begin{array}{l}\text { Adult/ } \\
\text { Immature }\end{array}$ & Acroceridae & $\begin{array}{l}\text { Ogcodes } \\
\text { rufoabdominalis Cole }\end{array}$ & Eason et al. 1967 \\
\hline & & $\begin{array}{c}\text { Pardosa saxatilis } \\
\text { (Hentz, 1844) }\end{array}$ & Immature & Acroceridae & $\begin{array}{l}\text { Ogcodes pallidipennis } \\
\text { (Loew) }\end{array}$ & Kaston 1937 \\
\hline
\end{tabular}


Table 1.-Continued.

\begin{tabular}{|c|c|c|c|c|c|c|}
\hline Host order & Host family & Arachnid host & Host stage & Fly family & $\begin{array}{c}\text { Fly parasitoid/ } \\
\text { parasite/predator }\end{array}$ & Reference \\
\hline \multirow[t]{15}{*}{ Araneae } & \multirow[t]{15}{*}{ Lycosidae } & $\begin{array}{r}\text { Pardosa sternalis } \\
\text { (Thorell, 1877) }\end{array}$ & $\begin{array}{l}\text { Adult/ } \\
\text { Immature }\end{array}$ & Acroceridae & Acrocera convexa Cole & Schlinger 1987 \\
\hline & & Pardosa sternalis & Immature & Acroceridae & $\begin{array}{l}\text { Ogcodes adaptatus } \\
\text { Schlinger }\end{array}$ & Schlinger 1960 \\
\hline & & Pardosa sternalis & Immature & Acroceridae & $\begin{array}{l}\text { Ogcodes eugonatus } \\
\text { (Loew) }\end{array}$ & Schlinger 1960 \\
\hline & & Pardosa sternalis & $\begin{array}{l}\text { Adult/ } \\
\text { Immature }\end{array}$ & Acroceridae & $\begin{array}{l}\text { Pterodontia misella } \\
\text { Osten Sacken }\end{array}$ & Schlinger 1987 \\
\hline & & $\begin{array}{l}\text { Pardosa tuoba } \\
\quad \text { Chamberlin, } 1919\end{array}$ & $\begin{array}{l}\text { Adult/ } \\
\text { Immature }\end{array}$ & Acroceridae & $\begin{array}{l}\text { Ogcodes adaptatus } \\
\text { Schlinger }\end{array}$ & Schlinger 1987 \\
\hline & & $\begin{array}{l}\text { Pardosa utahensis } \\
\text { Chamberlin, } 1919\end{array}$ & $\begin{array}{l}\text { Adult/ } \\
\text { Immature }\end{array}$ & Acroceridae & $\begin{array}{l}\text { Ogcodes } \\
\text { rufoabdominalis } \\
\text { Cole }\end{array}$ & Capelle 1966 \\
\hline & & $\begin{array}{c}\text { Pardosa vancouveri } \\
\text { Emerton, } 1917\end{array}$ & $\begin{array}{l}\text { Adult/ } \\
\text { Immature }\end{array}$ & Acroceridae & Acrocera convexa Cole & Schlinger 1987 \\
\hline & & $\begin{array}{l}\text { Pirata sedentarius } \\
\quad \text { Montgomery, } 1904\end{array}$ & $\begin{array}{l}\text { Adult/ } \\
\text { Immature }\end{array}$ & Acroceridae & $\begin{array}{l}\text { Ogcodes dispar } \\
\text { (Macquart) }\end{array}$ & Eason et al. 1967 \\
\hline & & $\begin{array}{l}\text { Schizocosa crassipes } \\
\quad \text { (Walckenaer, 1837) }\end{array}$ & $\begin{array}{l}\text { Adult/ } \\
\text { Immature }\end{array}$ & Acroceridae & $\begin{array}{l}\text { Acrocera fasciata } \\
\text { Wiedemann }\end{array}$ & $\begin{array}{l}\text { Montgomery } 1903 \text {, } \\
\text { Johnson } 1915\end{array}$ \\
\hline & & $\begin{array}{l}\text { Schizocosa ocreata } \\
\text { (Hentz, 1844) }\end{array}$ & $\begin{array}{l}\text { Adult/ } \\
\text { Immature }\end{array}$ & Acroceridae & $\begin{array}{l}\text { Acrocera fasciata } \\
\text { Wiedemann }\end{array}$ & $\begin{array}{c}\text { Montgomery } 1903 \text {, } \\
\text { Johnson } 1915\end{array}$ \\
\hline & & $\begin{array}{l}\text { Schizocosa rovneri } \\
\text { Uetz \& Dondale, } \\
1979\end{array}$ & $\begin{array}{l}\text { Adult/ } \\
\text { Immature }\end{array}$ & Acroceridae & Ogcodes borealis Cole & Cady et al. 1993 \\
\hline & & Schizocosa rovneri & $\begin{array}{l}\text { Adult/ } \\
\text { Immature }\end{array}$ & Acroceridae & $\begin{array}{l}\text { Ogcodes pallidipennis } \\
\text { (Loew) }\end{array}$ & Cady et al. 1993 \\
\hline & & $\begin{array}{l}\text { Tigrosa helluo } \\
\text { (Walckenaer, 1837) }\end{array}$ & $\begin{array}{l}\text { Adult/ } \\
\text { Immature }\end{array}$ & Acroceridae & $\begin{array}{l}\text { Ogcodes pallidipennis } \\
\text { (Loew) }\end{array}$ & Eason et al. 1967 \\
\hline & & $\begin{array}{l}\text { Trochosa hispanica } \\
\quad \text { Simon, } 1870\end{array}$ & $\begin{array}{l}\text { Adult/ } \\
\text { Immature }\end{array}$ & Acroceridae & $\begin{array}{l}\text { Ogcodes lautereri } \\
\text { Chvala }\end{array}$ & $\begin{array}{l}\text { Canzoneri \& Hansen } \\
1996\end{array}$ \\
\hline & & $\begin{array}{l}\text { Trochosa terricola } \\
\text { Thorell, } 1856\end{array}$ & $\begin{array}{l}\text { Adult/ } \\
\text { Immature }\end{array}$ & Acroceridae & $\begin{array}{l}\text { Pterodontia flavipes } \\
\text { Gray }\end{array}$ & King 1916 \\
\hline Araneae & Migidae & $\begin{array}{l}\text { Moggridgea crudeni } \\
\text { Hewitt, } 1913\end{array}$ & $\begin{array}{l}\text { Adult/ } \\
\text { Immature }\end{array}$ & Acroceridae & $\begin{array}{l}\text { Astomella capensis } \\
\text { Schlinger }\end{array}$ & Barraclough 1984 \\
\hline Araneae & Mimetidae & $\begin{array}{l}\text { Mimetus notius } \\
\quad \text { Chamberlin, } 1923\end{array}$ & Egg & Phoridae & $\begin{array}{l}\text { Phalacrotophora } \\
\text { epeirae (Brues) }\end{array}$ & Guarisco 2001 \\
\hline \multirow[t]{2}{*}{ Araneae } & \multirow[t]{2}{*}{ Miturgidae } & Griswoldia $\mathrm{sp}$. & Immature & Acroceridae & $\begin{array}{l}\text { Thyllis crassa } \\
\text { (Fabricius) }\end{array}$ & Schlinger 1987 \\
\hline & & Griswoldia sp. & $\begin{array}{l}\text { Adult/ } \\
\text { Immature }\end{array}$ & Acroceridae & Thyllis sp. & Schlinger 2003 \\
\hline \multirow[t]{4}{*}{ Araneae } & \multirow[t]{4}{*}{ Nephilidae } & $\begin{array}{l}\text { Nephila clavipes } \\
\quad \text { (Linnaeus, 1767) }\end{array}$ & Egg & Chloropidae & $\begin{array}{l}\text { Pseudogaurax higginsi } \\
\text { Sabrosky }\end{array}$ & Barnes et al. 1992 \\
\hline & & Nephila clavipes & Egg & Chloropidae & $\begin{array}{l}\text { Pseudogaurax } \\
\text { mexoculatus } \\
\text { Sabrosky }\end{array}$ & Barnes et al. 1992 \\
\hline & & $\begin{array}{r}\text { Nephila inaurata } \\
\text { (Vinson, 1863) }\end{array}$ & Egg & Chloropidae & $\begin{array}{l}\text { Pseudogaurax coyleae } \\
\text { Cogan }\end{array}$ & Cogan 1977 \\
\hline & & $\begin{array}{l}\text { Nephila pilipes } \\
\quad \text { (Fabricius, 1793) }\end{array}$ & Egg & Chloropidae & $\begin{array}{l}\text { Pseudogaurax seguyi } \\
\text { (Sabrosky) }\end{array}$ & Sabrosky 1990 \\
\hline Araneae & Oecobiidae & $\begin{array}{l}\text { Uroctea limbata (C. L. } \\
\text { Koch, 1843) }\end{array}$ & Egg & Bombyliidae & $\begin{array}{l}\text { Petrorossia feti } \\
\text { Zaitsev \& } \\
\text { Charykuliev }\end{array}$ & $\begin{array}{l}\text { Zaitzev \& } \\
\quad \text { Charykuliev } 1981\end{array}$ \\
\hline \multirow[t]{3}{*}{ Araneae } & \multirow[t]{3}{*}{ Oxyopidae } & $\begin{array}{r}\text { Oxyopes lineatus } \\
\text { Latreille, } 1806\end{array}$ & $\begin{array}{l}\text { Adult/ } \\
\text { Immature }\end{array}$ & Acroceridae & $\begin{array}{l}\text { Ogcodes fumatus } \\
\text { (Erichson) }\end{array}$ & Schlinger 1987 \\
\hline & & $\begin{array}{l}\text { Oxyopes salticus } \\
\text { Hentz, } 1845\end{array}$ & $\begin{array}{l}\text { Adult/ } \\
\text { Immature }\end{array}$ & Acroceridae & $\begin{array}{l}\text { Ogcodes dispar } \\
\text { (Macquart) }\end{array}$ & Eason et al. 1967 \\
\hline & & Oxyopes salticus & $\begin{array}{l}\text { Adult/ } \\
\text { Immature }\end{array}$ & Acroceridae & $\begin{array}{l}\text { Ogcodes eugonatus } \\
\text { (Loew) }\end{array}$ & Eason et al. 1967 \\
\hline Araneae & Philodromidae & $\begin{array}{l}\text { Philodromus aureolus } \\
\text { (Clerck, 1757) }\end{array}$ & Egg & Sarcophagidae & $\begin{array}{l}\text { Sarcophaga } \\
\text { sexpunctata } \\
\text { (Fabricius) }\end{array}$ & Auten 1925 \\
\hline
\end{tabular}


Table 1.-Continued.

\begin{tabular}{|c|c|c|c|c|c|c|}
\hline Host order & Host family & Arachnid host & Host stage & Fly family & $\begin{array}{c}\text { Fly parasitoid/ } \\
\text { parasite/predator }\end{array}$ & Reference \\
\hline \multirow[t]{4}{*}{ Araneae } & \multirow[t]{4}{*}{ Philodromidae } & $\begin{array}{l}\text { Philodromus cespitum } \\
\text { (Walckenaer, 1802) }\end{array}$ & Immature & Acroceridae & $\begin{array}{l}\text { Ogcodes fumatus } \\
\text { (Erichson) }\end{array}$ & $\begin{array}{l}\text { Kehlmaier et al. } \\
2012\end{array}$ \\
\hline & & Philodromus cespitum & Egg & Chloropidae & $\begin{array}{l}\text { Oscinella halterata } \\
\text { (Lamb) }\end{array}$ & Auten 1925 \\
\hline & & Philodromus cespitum & Egg & Sarcophagidae & $\begin{array}{l}\text { Sarcophaga } \\
\text { sexpunctata } \\
\text { (Fabricius) }\end{array}$ & Auten 1925 \\
\hline & & Philodromus sp. & Immature & Acroceridae & $\begin{array}{l}\text { Ogcodes adaptatus } \\
\text { Schlinger }\end{array}$ & Schlinger 1960 \\
\hline Araneae & Phyxelididae & $\begin{array}{l}\text { Ambohima sublima } \\
\text { Griswold, } 1990\end{array}$ & Immature & Acroceridae & Thyllis sp. & Schlinger 2003 \\
\hline Araneae & Plectreuridae & Kibramoa sp. & $\begin{array}{l}\text { Adult/ } \\
\text { Immature }\end{array}$ & Acroceridae & $\begin{array}{l}\text { Acrocera arizonensis } \\
\text { Cole }\end{array}$ & Schlinger 1987 \\
\hline \multirow[t]{22}{*}{ Araneae } & \multirow[t]{22}{*}{ Salticidae } & $\begin{array}{l}\text { Aelurillus v-insignitus } \\
\text { (Clerck, 1757) }\end{array}$ & Adult & Acroceridae & $\begin{array}{l}\text { Ogcodes pallipes } \\
\text { Latreille }\end{array}$ & Millot 1938 \\
\hline & & $\begin{array}{l}\text { Aelurillus v-insignitus } \\
\text { (Clerck) }\end{array}$ & $\begin{array}{l}\text { Adult/ } \\
\text { Immature }\end{array}$ & Acroceridae & $\begin{array}{l}\text { Ogcodes varius } \\
\text { Latreille }\end{array}$ & Séguy 1926 \\
\hline & & Cobanus sp. & $\begin{array}{l}\text { Aduit/ } \\
\text { Immature }\end{array}$ & Acroceridae & Terphis sp. & Schlinger 1987 \\
\hline & & $\begin{array}{l}\text { Cosmophasis } \\
\text { bitaeniata } \\
\text { (Keyserling, 1882) }\end{array}$ & $\begin{array}{l}\text { Adult/ } \\
\text { Immature }\end{array}$ & Acroceridae & $\begin{array}{l}\text { Ogcodes basalis } \\
\text { (Walker) }\end{array}$ & Schlinger 1987 \\
\hline & & $\begin{array}{c}\text { Cosmophasis } \\
\text { bitaeniata }\end{array}$ & $\begin{array}{l}\text { Adult/ } \\
\text { Immature }\end{array}$ & Acroceridae & $\begin{array}{l}\text { Ogcodes doddi } \\
\text { Wandolleck }\end{array}$ & $\begin{array}{l}\text { Wandolleck 1906, } \\
\text { Dodd } 1906\end{array}$ \\
\hline & & $\begin{array}{l}\text { Eris militaris (Hentz, } \\
1845 \text { ) }\end{array}$ & Immature & Acroceridae & Acrocera sp. & $\begin{array}{l}\text { Larrivée \& Borkent } \\
2009\end{array}$ \\
\hline & & $\begin{array}{l}\text { Evarcha jucunda } \\
\quad \text { (Lucas, 1846) }\end{array}$ & Immature & Acroceridae & $\begin{array}{l}\text { Ogcodes reginae } \\
\text { Trojan }\end{array}$ & $\begin{array}{l}\text { Kehlmaier \& } \\
\text { Almeida } 2014\end{array}$ \\
\hline & & $\begin{array}{l}\text { Habronattus hallani } \\
\text { (Richman, 1973) }\end{array}$ & $\begin{array}{l}\text { Adult/ } \\
\text { Immature }\end{array}$ & Acroceridae & $\begin{array}{l}\text { Ogcodes eugonatus } \\
\text { (Loew) }\end{array}$ & Schlinger 1987 \\
\hline & & Heliophanus sp. & Immature & Acroceridae & $\begin{array}{l}\text { Ogcodes pallipes } \\
\text { Latreille }\end{array}$ & Millot 1938 \\
\hline & & Heliophanus sp. & Immature & Acroceridae & $\begin{array}{l}\text { Ogcodes zonatus } \\
\text { Erichson }\end{array}$ & Millot 1938 \\
\hline & & Lyssomaninae gen. sp. & $\begin{array}{l}\text { Adult/ } \\
\text { Immature }\end{array}$ & Acroceridae & $\begin{array}{l}\text { Ogcodes guttatus } \\
\text { (Costa) }\end{array}$ & Schlinger 1987 \\
\hline & & $\begin{array}{l}\text { Metaphidippus manni } \\
\text { (Peckham \& } \\
\text { Peckham, } 1901\end{array}$ & $\begin{array}{l}\text { Adult/ } \\
\text { Immature }\end{array}$ & Acroceridae & $\begin{array}{l}\text { Pterodontia vix } \\
\text { Townsend }\end{array}$ & Schlinger 1987 \\
\hline & & Metaphidippus sp. & $\begin{array}{l}\text { Adult/ } \\
\text { Immature }\end{array}$ & Acroceridae & $\begin{array}{c}\text { Acrocera bulla } \\
\text { Westwood }\end{array}$ & Schlinger 1987 \\
\hline & & Metaphidippus sp. & $\begin{array}{l}\text { Adult/ } \\
\text { Immature }\end{array}$ & Acroceridae & $\begin{array}{l}\text { Ogcodes boharti } \\
\text { Schlinger }\end{array}$ & Schlinger 1987 \\
\hline & & $\begin{array}{l}\text { Pelegrina aeneola } \\
\quad \text { (Curtis, 1892) }\end{array}$ & $\begin{array}{l}\text { Adult/ } \\
\text { Immature }\end{array}$ & Acroceridae & $\begin{array}{l}\text { Acrocera bulla } \\
\text { Westwood }\end{array}$ & Beckwith et al. 1987 \\
\hline & & Pelegrina aeneola & $\begin{array}{l}\text { Adult/ } \\
\text { Immature }\end{array}$ & Acroceridae & $\begin{array}{l}\text { Ogcodes boharti } \\
\text { Schlinger }\end{array}$ & Beckwith et al. 1987 \\
\hline & & Pelegrina aeneola & $\begin{array}{l}\text { Adult/ } \\
\text { Immature }\end{array}$ & Acroceridae & Ogcodes borealis Cole & Schlinger 1987 \\
\hline & & $\begin{array}{l}\text { Pelegrina proterva } \\
\quad \text { (Walckenaer, 1837) }\end{array}$ & Immature & Acroceridae & $\begin{array}{l}\text { Ogcodes eugonatus } \\
\text { Loew }\end{array}$ & $\begin{array}{l}\text { Larrivée \& Borkent } \\
2009\end{array}$ \\
\hline & & Pelegrina proterva & Immature & Acroceridae & $\begin{array}{l}\text { Ogcodes melampus } \\
\text { Loew }\end{array}$ & $\begin{array}{l}\text { Larrivée \& Borkent } \\
2009\end{array}$ \\
\hline & & $\begin{array}{l}\text { Phidippus ardens } \\
\text { Peckham \& } \\
\text { Peckham, } 1901\end{array}$ & $\begin{array}{l}\text { Adult/ } \\
\text { Immature }\end{array}$ & Acroceridae & $\begin{array}{l}\text { Ogcodes boharti } \\
\text { Schlinger }\end{array}$ & Schlinger 1987 \\
\hline & & $\begin{array}{l}\text { Phidippus audax } \\
\text { (Hentz, 1845) }\end{array}$ & Egg & Phoridae & $\begin{array}{l}\text { Phalacrotophora } \\
\text { epeirae (Brues) }\end{array}$ & Jones 1940 \\
\hline & & $\begin{array}{l}\text { Phidippus comatus } \\
\text { Peckham \& } \\
\text { Peckham, } 1901\end{array}$ & $\begin{array}{l}\text { Adult/ } \\
\text { Immature }\end{array}$ & Acroceridae & $\begin{array}{l}\text { Ogcodes boharti } \\
\text { Schlinger }\end{array}$ & Schlinger 1987 \\
\hline
\end{tabular}


Table 1.-Continued.

\begin{tabular}{|c|c|c|c|c|c|c|}
\hline Host order & Host family & Arachnid host & Host stage & Fly family & $\begin{array}{l}\text { Fly parasitoid/ } \\
\text { parasite/predator }\end{array}$ & Reference \\
\hline \multirow[t]{11}{*}{ Araneae } & \multirow[t]{11}{*}{ Salticidae } & $\begin{array}{l}\text { Phidippus johnsoni } \\
\text { (Peckham \& } \\
\text { Peckham, 1883) }\end{array}$ & $\begin{array}{l}\text { Adult/ } \\
\text { Immature }\end{array}$ & Acroceridae & $\begin{array}{l}\text { Ogcodes adaptatus } \\
\text { Schlinger }\end{array}$ & Schlinger 1987 \\
\hline & & Phidippus johnsoni & $\begin{array}{l}\text { Adult/ } \\
\text { Immature }\end{array}$ & Acroceridae & $\begin{array}{l}\text { Ogcodes boharti } \\
\text { Schlinger }\end{array}$ & Schlinger 1987 \\
\hline & & Phidippus johnsoni & $\begin{array}{l}\text { Adult/. } \\
\text { Immature }\end{array}$ & Acroceridae & $\begin{array}{l}\text { Ogcodes eugonatus } \\
\text { (Loew) }\end{array}$ & Schlinger 1987 \\
\hline & & $\begin{array}{l}\text { Phidippus } \\
\text { octopunctatus } \\
\text { (Peckham \& } \\
\text { Peckham, 1883) }\end{array}$ & Egg & Sarcophagidae & $\begin{array}{l}\text { Sarcophaga davidsonii } \\
\text { Coquillett }\end{array}$ & $\begin{array}{l}\text { Coquillet 1892; } \\
\text { Davidson } 1896\end{array}$ \\
\hline & & $\begin{array}{l}\text { Phidippus princeps } \\
\text { (Peckham \& } \\
\text { Peckham, 1883) }\end{array}$ & $\begin{array}{l}\text { Adult/ } \\
\text { Immature }\end{array}$ & Acroceridae & $\begin{array}{l}\text { Ogcodes eugonatus } \\
\text { (Loew) }\end{array}$ & Schlinger 1987 \\
\hline & & $\begin{array}{l}\text { Phidippus regius C. L. } \\
\text { Koch, } 1846\end{array}$ & Egg & Phoridae & $\begin{array}{l}\text { Phalacrotophora } \\
\text { epeirae (Brues) }\end{array}$ & Manuel 1984 \\
\hline & & $\begin{array}{l}\text { Phidippus rimator } \\
\text { nom. dub. } \\
\text { (Walckenaer, 1837) }\end{array}$ & $\begin{array}{l}\text { Adult/ } \\
\text { Immature }\end{array}$ & Acroceridae & $\begin{array}{l}\text { Ogcodes eugonatus } \\
\text { (Loew) }\end{array}$ & Schlinger 1987 \\
\hline & & Phidippus sp. & $\begin{array}{l}\text { Adult/ } \\
\text { Immature }\end{array}$ & Acroceridae & Acrocera sp. & Schlinger 1987 \\
\hline & & $\begin{array}{c}\text { Phlegra fasciata } \\
\text { (Hahn, 1826) }\end{array}$ & Immature & Acroceridae & $\begin{array}{l}\text { Ogcodes pallipes } \\
\text { Latreille }\end{array}$ & Millot 1938 \\
\hline & & Sassacus sp. & $\begin{array}{l}\text { Adult/ } \\
\text { Immature }\end{array}$ & Acroceridae & $\begin{array}{l}\text { Ogcodes eugonatus } \\
\text { (Loew) }\end{array}$ & Schlinger 1987 \\
\hline & & Sidusa sp. & $\begin{array}{l}\text { Adult/ } \\
\text { Immature }\end{array}$ & Acroceridae & Terphis sp. & Schlinger 2003 \\
\hline Araneae & Segestriidae & $\begin{array}{l}\text { Ariadna maxima } \\
\text { (Nicolet, 1849) }\end{array}$ & $\begin{array}{l}\text { Adult/ } \\
\text { Immature }\end{array}$ & Acroceridae & $\begin{array}{l}\text { Carvalhoa } \\
\text { appendiculata } \\
\text { Philippi }\end{array}$ & Schlinger 1987 \\
\hline \multirow[t]{3}{*}{ Araneae } & \multirow[t]{3}{*}{ Tetragnathidae } & $\begin{array}{l}\text { Meta menardi } \\
\quad(\text { Latreille, 1804) }\end{array}$ & Egg & Phoridae & $\begin{array}{l}\text { Megaselia } \\
\quad \text { melanocephala } \\
\quad \text { (Roser) }\end{array}$ & $\begin{array}{l}\text { Decou-Burghele } \\
1961\end{array}$ \\
\hline & & Tetragnatha $\mathrm{sp}$. & Egg & Chloropidae & $\begin{array}{l}\text { Pseudogaurax } \\
\text { silbergliedi Sabrosky }\end{array}$ & Sabrosky 1990 \\
\hline & & Tetragnatha sp. & Egg & Chloropidae & Siphonella sp. & Kintner 1935 \\
\hline \multirow[t]{8}{*}{ Araneae } & \multirow[t]{8}{*}{ Theraphosidae } & $\begin{array}{l}\text { Acanthoscurria } \\
\text { sternalis Pocock, } \\
1903\end{array}$ & Immature & Acroceridae & $\begin{array}{l}\text { Exetasis jujuyensis } \\
\text { Gillung }\end{array}$ & Barneche et al. 2013 \\
\hline & & $\begin{array}{l}\text { Aphonopelma duplex } \\
\text { (Chamberlin, 1925) }\end{array}$ & $\begin{array}{l}\text { Adult/ } \\
\text { Immature }\end{array}$ & Acroceridae & Ocnaea sp. & $\begin{array}{l}\text { Alagon \& Odell } \\
2004\end{array}$ \\
\hline & & $\begin{array}{l}\text { Aphonopelma hentzi } \\
\text { (Girard, 1852) }\end{array}$ & $\begin{array}{l}\text { Adult/ } \\
\text { Immature }\end{array}$ & Acroceridae & $\begin{array}{l}\text { Lasia purpurata } \\
\text { Bequaert }\end{array}$ & $\begin{array}{l}\text { Baerg 1958; Eason } \\
\text { et al. } 1967\end{array}$ \\
\hline & & Aphonopelma sp. & $\begin{array}{l}\text { Adult/ } \\
\text { Immature }\end{array}$ & Acroceridae & Ocnaea sp. & Schlinger 1987 \\
\hline & & Chaetopelma sp. & $\begin{array}{l}\text { Adult/ } \\
\text { Immature }\end{array}$ & Acroceridae & $\begin{array}{l}\text { Astomella gravis } \\
\text { Erichson }\end{array}$ & Schlinger 1987 \\
\hline & & $\begin{array}{l}\text { Grammostola actaeon } \\
\text { (Pocock, 1903) }\end{array}$ & Adult & Acroceridae & Exetasis sp. & Vellard 1934 \\
\hline & & $\begin{array}{l}\text { Lasiodora klugi (C. L. } \\
\text { Koch, 1841) }\end{array}$ & Immature & Acroceridae & $\begin{array}{l}\text { Exetasis eickstedtae } \\
\text { Schlinger }\end{array}$ & $\begin{array}{l}\text { Eickstedt } 1971 ; \\
\text { Schlinger } 1972\end{array}$ \\
\hline & & $\begin{array}{l}\text { Phrixotrichus scrofa } \\
\text { (Molina, 1788) }\end{array}$ & Immature & Acroceridae & $\begin{array}{l}\text { Arrhynchus maculatus } \\
\text { Schlinger }\end{array}$ & Schlinger 1968 \\
\hline \multirow[t]{4}{*}{ Araneae } & \multirow[t]{4}{*}{ Theridiidae } & $\begin{array}{l}\text { Enoplognatha ovata } \\
\text { (Clerck, 1757) }\end{array}$ & $\begin{array}{l}\text { Adult/ } \\
\text { Immature }\end{array}$ & Acroceridae & $\begin{array}{l}\text { Ogcodes gibbosus } \\
\text { (Linnaeus) }\end{array}$ & Pichka 1977 \\
\hline & & Enoplognatha sp. & Egg & Phoridae & $\begin{array}{l}\text { Megaselia tenebricola } \\
\text { Schmitz }\end{array}$ & Evans 1969 \\
\hline & & $\begin{array}{l}\text { Enoplognatha } \mathrm{sp} . \text { or } \\
\quad \text { Robertus } \mathrm{sp} .\end{array}$ & Egg & Phoridae & $\begin{array}{l}\text { Megaselia angusta } \\
\text { Wood }\end{array}$ & $\begin{array}{l}\text { Disney \& Evans } \\
\text { 1980; Disney } 1999\end{array}$ \\
\hline & & $\begin{array}{l}\text { Enoplognatha } \mathrm{sp} . \text { or } \\
\quad \text { Robertus } \mathrm{sp} .\end{array}$ & Egg & Phoridae & $\begin{array}{l}\text { Megaselia longifurca } \\
\text { (Lundbeck) }\end{array}$ & $\begin{array}{l}\text { Disney \& Evans } \\
\text { 1980; Disney } 1999\end{array}$ \\
\hline
\end{tabular}


Table 1.-Continued.

\begin{tabular}{|c|c|c|c|c|c|c|}
\hline Host order & Host family & Arachnid host & Host stage & Fly family & $\begin{array}{l}\text { Fly parasitoid/ } \\
\text { parasite/predator }\end{array}$ & Reference \\
\hline \multirow[t]{8}{*}{ Araneae } & \multirow[t]{8}{*}{ Theridiidae } & $\begin{array}{l}\text { Latrodectus } \\
\text { geometricus C.L. } \\
\text { Koch, } 1841\end{array}$ & Egg & Chloropidae & $\begin{array}{l}\text { Pseudogaurax signatus } \\
\text { (Loew) }\end{array}$ & Vetter et al. 2012 \\
\hline & & $\begin{array}{l}\text { Latrodectus hesperus } \\
\text { Chamberlin \& Ivie, } \\
1935\end{array}$ & Egg & Chloropidae & $\begin{array}{l}\text { Pseudogaurax signatus } \\
\text { (Loew) }\end{array}$ & Vetter et al. 2012 \\
\hline & & $\begin{array}{l}\text { Latrodectus mactans } \\
\text { (Fabricius, 1775) }\end{array}$ & Egg & Phoridae & $\begin{array}{l}\text { Apocephalus borealis } \\
\text { Brues }\end{array}$ & Disney 1994 \\
\hline & & Latrodectus mactans & Egg & Chloropidae & $\begin{array}{l}\text { Pseudogaurax signatus } \\
\text { (Loew) }\end{array}$ & Davidson 1896 \\
\hline & & Latrodectus mactans & Egg & Chloropidae & Pseudogaurax sp. & Baerg 1959 \\
\hline & & $\begin{array}{l}\text { Parasteatoda } \\
\text { tepidariorum }(\mathrm{L} . \\
\text { Koch, 1841) }\end{array}$ & Egg & Chloropidae & $\begin{array}{l}\text { Pseudogaurax signatus } \\
\text { (Loew) }\end{array}$ & $\begin{array}{l}\text { Kaston \& Jenks } \\
1937\end{array}$ \\
\hline & & Robertus sp. & Egg & Phoridae & $\begin{array}{l}\text { Megaselia tenebricola } \\
\text { Schmitz }\end{array}$ & $\begin{array}{l}\text { Disney \& Evans } \\
1980\end{array}$ \\
\hline & & $\begin{array}{l}\text { Steatoda palomara } \\
\text { Chamberlin \& Ivie, } \\
1935\end{array}$ & Immature & Acroceridae & $\begin{array}{l}\text { Ogcodes pallidipennis } \\
\text { (Loew) }\end{array}$ & Schlinger 1960 \\
\hline \multirow[t]{11}{*}{ Araneae } & \multirow[t]{11}{*}{ Thomisidae } & Diaea $\mathrm{sp}$ & $\begin{array}{l}\text { Adult/ } \\
\text { Immature }\end{array}$ & Acroceridae & $\begin{array}{l}\text { Ogcodes nitens } \\
\text { (Hutton) }\end{array}$ & Schlinger 1987 \\
\hline & & Mecaphesa sp. & Immature & Acroceridae & $\begin{array}{l}\text { Ogcodes eugonatus } \\
\text { Loew }\end{array}$ & $\begin{array}{l}\text { Cokendolpher et al. } \\
1979\end{array}$ \\
\hline & & $\begin{array}{l}\text { Misumena vatia } \\
\text { (Clerck, 1757) }\end{array}$ & $\begin{array}{l}\text { Adult/ } \\
\text { Immature }\end{array}$ & Acroceridae & $\begin{array}{l}\text { Ogcodes fumatus } \\
\text { (Erichson) }\end{array}$ & de Jong et al. 2000 \\
\hline & & $\begin{array}{l}\text { Thomisus onustus } \\
\text { (Walckenaer, 1806) }\end{array}$ & $\begin{array}{l}\text { Adult/ } \\
\text { Immature }\end{array}$ & Acroceridae & $\begin{array}{l}\text { Ogcodes fumatus } \\
\text { (Erichson) }\end{array}$ & de Jong et al. 2000 \\
\hline & & $\begin{array}{l}\text { Xysticus cunctator } \\
\text { Thorell, } 1877\end{array}$ & Immature & Acroceridae & $\begin{array}{l}\text { Ogcodes adaptatus } \\
\text { Schlinger }\end{array}$ & Schlinger 1960 \\
\hline & & Xysticus cunctator & Immature & Acroceridae & $\begin{array}{l}\text { Ogcodes melampus } \\
\text { (Loew) }\end{array}$ & Schlinger 1960 \\
\hline & & $\begin{array}{l}\text { Xysticus luctuosus } \\
\text { (Blackwall, 1836) }\end{array}$ & $\begin{array}{l}\text { Adult/ } \\
\text { Immature }\end{array}$ & Acroceridae & $\begin{array}{l}\text { Ogcodes pallipes } \\
\text { Latreille }\end{array}$ & Trojan 1956 \\
\hline & & $\begin{array}{l}\text { Xysticus montanensis } \\
\text { Keyserling1887 }\end{array}$ & Immature & Acroceridae & Ogcodes borealis Cole & Schlinger 1960 \\
\hline & & Xysticus montanensis & Immature & Acroceridae & $\begin{array}{l}\text { Ogcodes pallidipennis } \\
\text { (Loew) }\end{array}$ & Schlinger 1960 \\
\hline & & Xysticus sp. & $\begin{array}{l}\text { Adult/ } \\
\text { Immature }\end{array}$ & Acroceridae & $\begin{array}{l}\text { Ogcodes eugonatus } \\
\text { (Loew) }\end{array}$ & Schlinger 1987 \\
\hline & & Undetermined genus & Egg & Drosophilidae & $\begin{array}{l}\text { Scaptomyza } \\
\text { (Titanochaeta) } 11 \\
\text { spp. }\end{array}$ & $\begin{array}{l}\text { Wirth 1952; Hardy } \\
\text { 1965; Lapoint et } \\
\text { al. } 2013\end{array}$ \\
\hline Araneae & Trachelidae & $\begin{array}{l}\text { Trachelas mexicanus } \\
\text { Banks, } 1898\end{array}$ & $\begin{array}{l}\text { Adult/ } \\
\text { Immature }\end{array}$ & Acroceridae & $\begin{array}{l}\text { Ogcodes pallidipennis } \\
\text { (Loew) }\end{array}$ & Schlinger 1987 \\
\hline Araneae & Unknown & Undetermined genus & Egg & Phoridae & $\begin{array}{l}\text { Megaselia oviaraneae } \\
\text { Disney }\end{array}$ & Disney 1999 \\
\hline \multirow[t]{2}{*}{ Scorpiones } & \multirow[t]{2}{*}{ Buthidae } & $\begin{array}{l}\text { Centruroides } \\
\quad \text { margaritatus } \\
\text { (Gervais, 1841) }\end{array}$ & $\begin{array}{l}\text { Adult/ } \\
\text { Immature }\end{array}$ & Sarcophagidae & $\begin{array}{l}\text { Sarcodexia } \\
\text { sternodontis } \\
\text { Townsend }\end{array}$ & Townsend 1893 \\
\hline & & $\begin{array}{l}\text { Mesobuthus martensii } \\
\text { (Karsch, 1879) }\end{array}$ & $\begin{array}{l}\text { Adult/ } \\
\text { Immature }\end{array}$ & Sarcophagidae & $\begin{array}{l}\text { Sarcophaga dux } \\
\text { Thomson }\end{array}$ & Shi et al. 2015 \\
\hline \multirow[t]{2}{*}{ Scorpiones } & \multirow[t]{2}{*}{ Vaejovidae } & $\begin{array}{l}\text { Anuroctonus } \\
\text { phaiodactylus } \\
\text { (Wood, 1863) }\end{array}$ & Adult & Tachinidae & $\begin{array}{l}\text { Spilochaetosoma } \\
\quad \text { californicum } \text { Smith }\end{array}$ & Williams et al. 1994 \\
\hline & & $\begin{array}{l}\text { Vaejovis spinigerus } \\
\text { (Wood, 1863) }\end{array}$ & $\begin{array}{l}\text { Adult/ } \\
\text { Immature }\end{array}$ & Tachinidae & $\begin{array}{l}\text { Spilochaetosoma } \\
\text { californicum } \text { Smith }\end{array}$ & Williams et al. 1994 \\
\hline Trombidiformes & Anystidae & Undetermined genus & $\begin{array}{l}\text { Adult/ } \\
\text { Immature }\end{array}$ & Acroceridae & Undetermined genus & $\begin{array}{l}\text { Kerr \& Winterton } \\
2008\end{array}$ \\
\hline Trombidiformes & Erythraeidae & Abrolophus sp. & $\begin{array}{l}\text { Adult/ } \\
\text { Immature }\end{array}$ & Acroceridae & $\begin{array}{l}\text { Pterodontia flavipes } \\
\text { Gray }\end{array}$ & Sferra 1986 \\
\hline Trombidiformes & Trombidiidae & Podothrombium sp. & $\begin{array}{l}\text { Adult/ } \\
\text { Immature }\end{array}$ & Acroceridae & $\begin{array}{l}\text { Pterodontia flavipes } \\
\text { Gray }\end{array}$ & Sferra 1986 \\
\hline
\end{tabular}


and deeper investigation is needed in order to verify whether the species in question are predators, parasitoids, or both.

In general, there is still much to be learned about the interactions between Diptera and the arachnids they attack. Many more large rearing studies of spiders and other arachnids would be the best approach to fill this gap in knowledge, as the majority of reports of natural enemies are the result of individual incidental rearings. Unfortunately, there are still large numbers of arachnid species that we know almost nothing about in terms of their habits, habitats, mating behavior and natural enemies. This lack of basic natural history information seriously limits the ability of arachnologists and dipterists to address these issues, though this problem is not limited to these groups or questions (Tewksbury et al. 2014; Barrows et al 2016). It is imperative that we as biologists spend time observing our chosen study organisms in their natural environment, if we are to have any hope of both discovering and understanding the ecological web of organisms that covers our planet.

\section{ACKNOWLEDGMENTS}

The authors thank Rick Vetter for his invitation to write this review for the Journal of Arachnology, which motivated us to finally put all of this information into one place. We also thank Dr. Steve Marshall and an anonymous reviewer for their comments on a previous version of the manuscript, as well as J.R. Maxwell, K. Reddick, J. Honaker and Dr. A. Alagon for allowing us to use their images in the figures.

\section{LITERATURE CITED}

Adler, P.H., S.R. Reitz \& C.N. Watson. 1997. Distribution and abundance of Eulonchus marialiciae (Diptera: Acroceridae). Entomological News 108:190-192.

Alagon, A. \& G. Odell. 2004. Acrocerid parasites of Mexican tarantulas. Forum Magazine of the American Tarantula Society 13:57.

Allard, C. \& M.W. Robertson. 2003. Nematode and dipteran endoparasites of the wolf spider Pardosa milvina (Araneae, Lycosidae). Journal of Arachnology 31:139-141.

Austin, A.D. 1985. The function of spider egg sacs in relation to parasitoids and predators, with special reference to the Australian fauna. Journal of Natural History 19:359-376.

Auten, M. 1925. Insects associated with spider nests. Annals of the Entomological Society of America 18:240-250.

Baerg, W.J. 1958. The Tarantula. University of Kansas Press, Lawrence.

Baerg, W.J. 1959. The black widow and five other venomous spiders. University of Arkansas (Fayetteville) Agricultural Experiment Station Bulletin 608:1-43.

Barnard, C.J. 1984. The evolution of food-scrounging strategies within and between species. Pp. 95-126. In Producers and Scroungers: Strategies of Exploitation and Parasitism.s (C.J. Barnard, ed.). Croom-Helm, London.

Barneche, J.A., J.P. Gillung \& A. Gonzalez. 2013. Description and host interactions of a new species of Exetasis Walker (Diptera: Acroceridae), with a key to species of the genus. Zootaxa 3664:525-536.

Barnes, J.K., L.E. Higgins \& C.W. Sabrosky. 1992. Life histories of Pseudogaurax species (Diptera: Chloropidae), descriptions of two new species, and ecology of Nephila clavipes (Linnaeus) (Araneae: Tetragnathidae) egg predation. Journal of Natural History 26:823834.
Barraclough, D.A. 1984. Review of some Afrotropical Acroceridae, with descriptions of eight new species from South Africa (Diptera: Brachycera). Journal of the Entomological Society of Southern Africa 47:45-66.

Barraclough, D.A. \& W. Croucamp. 1997. A new South African species of Ogcodes Latreille (Diptera: Acroceridae) reared from a sac spider of the genus Cheiracanthium Koch (Miturgidae). Annals of the Natal Museum 38:55-60.

Barrows, C.W., M.L. Murphy-Mariscal \& R.R. Hernandez. 2016. At a crossroads: the nature of natural history in the twenty-first century. BioScience 66:592-599.

Beckwith, R.C., R.R. Mason \& H.G. Paul. 1987. Parasitism of salticid spiders in Oregon by two species of Acroceridae (Diptera). Pan-Pacific Entomologist 63:352.

Bertkau, F. 1880. Verzeichniss der bisher bei Bonn beobachteten Spinnen. Verhandlungen des Naturhistorischen Vereines der preussischen Rheinlande und Westfalens 37. Kommission bei M. Cohen, Bonn.

Borkent, C.J. \& E.I. Schlinger. 2008. Pollen loads and diversity on bodies of Eulonchus tristis (Diptera: Acroceridae); implications for pollination and flower visitation. The Canadian Entomologist 140:257-264.

Brake, I. 2000. Phylogenetic systematics of the Milichiidae (Diptera, Schizophora). Entomologica Escandinavica, Supplemment 57:1120.

Brake, I. \& M. von Tschirnhaus. 2010. Stomosis arachnophila sp. n., a new kleptoparasitic species of freeloader flies (Diptera, Milichiidae). Zookeys 50:91-96.

Brauer, F. 1869. Beitrag zur Biologie der Acroceriden. Verhandlungen der Zoologisch-Botanischen Gesellschaft Wien 19:737-740.

Brignoli, P.M. 1976. Ragni d'Italia 26. Su di una nuova Malthonica di Sicilia parassitata da un dittero acroceride (Araneae, Agelenidae; Diptera, Acroceridae). Bollettino dell'Associazione Romana di Entomologia 30:30-35.

Brockmann, H.J. \& C.J. Barnard. 1979. Kleptoparasitism in birds. Animal Behaviour 27:487-514.

Brues, C.T. 1902. Notes on the larvae of some Texan Diptera. Psyche 9:351-354.

Brues, C.T. 1903. A monograph of the North American Phoridae. Transactions of the American Entomological Society 29:331-404.

Cady, A., R. Leech, L. Sorkin, G. Stratton \& M. Caldwell. 1993. Acrocerid (Insecta: Diptera) life histories, behaviors, host spiders (Arachnida: Araneida), and distributional records. Canadian Entomologist 125:931-944.

Cantrell, B.K. 1986. Notes on the taxonomy and biology of species of Parasarcophaga Johnston \& Tiegs and Baranovisca Lopes (Diptera: Sarcophagidae) associated with spiders in eastern Australia. Australian Entomological Magazine 13:1-10.

Canzoneri, S. \& H. Hansen. 1996. Prima segnalazione di Ogcodes lautereri Chvala, 1980 (Diptera: Acroceridae), ospite di Trochose hispanica (Simon, 1870) (Araneae: Lycosidae), in Italia. Societa Veneziana di Scienze Naturali Lavori 21:105.

Capelle, K.J. 1966. Observations on the life history of Ogcodes rufoabdominalis in Northern Utah (Diptera: Acroceridae). Journal of the Kansas Entomological Society 39:641-649.

Carvalho, R. \& I.C. Machado. 2006. Rodriguezia bahiensis Rchb. f.: biologia floral, polinizadores e primeiro registro de polinização por moscas Acroceridae em Orchidaceae. Revista Brasileira de Botânica 29:461-470.

Chapin, K.J. \& E.A. Hebets. 2016. The behavioral ecology of amblypygids. Journal of Arachnology 44:1-14.

Clastrier, J. \& J. Legrand. 1991. Forcipomyia (Trichohelea) araneivora n. sp. ectoparasite d'une araignee habitant les Monts Nimba en Guinee (Diptera, Ceratopogonidae; Araneae, Araneidae). Revue Francaise d'Entomologie (Nouvelle Serie) 13:155-158. 
Clausen, C.P. 1940. Entomophagous Insects. McGraw Hill Publishing Co., London.

Cogan, B.H. 1977. The Malagasy species of Pseudogaurax Malloch (Diptera: Chloropidae), and notes on some African species. Annals of the Natal Museum 23:117-127.

Cokendolpher, J.C., N.V. Horner \& D.T. Jennings. 1979. Crab Spiders of North-Central Texas (Araneae: Philodromidae and Thomisidae). Journal of the Kansas Entomological Society 52:723-734.

Cole, F.R. 1969. The Flies of Western North America. University of California Press, Berkeley \& Los Angeles.

Coquillett, D.W. 1892. The dipterous parasite of Melanoplus devastator in California. Insect Life 5:24.

Coquillett, D.W. 1898. Notes and descriptions of Oscinidae. Journal of the New York Entomological Society 6:44-49.

Coyle, F.A. 1971. Systematics and natural history of the mygalomorph spider genus Antrodiaetus and related genera (Araneae Antrodiaetidae). Bulletin of the Museum of Comparative Zoology 141:269-402.

Coyle, F.A. \& W.R. Icenogle. 1994. Natural history of the Californian trapdoor spider genus Aliatypus (Araneae, Antrodiaetidae). Journal of Arachnology 22:225-255.

Davidson, A. 1894. Concerning spider-egg parasites. Insect Life 6:268-269.

Davidson, A. 1896. Parasites of spider eggs. Entomological News 7:319-320.

de Armas, L.F. \& G.A. Garcia 1986. Depredadores y parasitoides de Argiope trifasciata (Araneae: Araneidae) en el sur de La Habana. Ciencias Biologicas Academia de Ciencias de Cuba 16: 114-117.

de Jong, H., A.P. Noordam \& T. Zeegers. 2000. The Acroceridae (Diptera) of The Netherlands. Entomologische Berichten 60:171179.

Decou-Burghele, A. 1961. Sur la biologie de Megaselia melanocephala von Roser, phoride parasite des cocons de Meta menardi Latr. Annales du Laboratoire Souterrain, Hans-sur-Lesse 11:16-22.

Dennis, D.S., R.J. Lavigne \& J.G. Dennis. 2012. Spiders (Araneae) as prey of robber flies (Diptera: Asilidae). Journal of the Entomological Research Society 14:65-76.

Disney, R.H.L. 1982. Megaselia argiopephaga n.sp. (Diptera: Phoridae), an Oriental scuttlefly whose larvae feed on spider eggs. Entomologica Scandinavica 13:321-324.

Disney, R.H.L. 1994. Scuttle Flies: The Phoridae. Chapman \& Hall, London.

Disney, R.H.L. 1999. A troublesome sibling species complex of scuttle flies (Diptera: Phoridae) revisited. Journal of Natural History 33:1159-1216.

Disney, R.H.L. \& R.E. Evans. 1980. Phoridae (Diptera) whose larvae feed on eggs of spiders (Araneida). Entomologist's Monthly Magazine 115:21-22.

Dodd, F.P. 1906. Notes upon some remarkable parasitic insects from North Queensland. Transactions of the Entomological Society of London 54:119-132.

Duffey, E. 2000. Rare dipteran parasite of a lycosid spider. British Arachnological Society Newsletter 87:3.

Dumbleton, L.J. 1940. Oncodes brunneus Hutton: a dipterous spider parasite. New Zealand Journal of Science and Technology (A) 22:97-102.

Eason, R.R. 1966. Life history and behavior of Pardosa lapidicina Emerton (Araneida: Lycosidae). Unpublished thesis, University of Arkansas.

Eason, R.R., W.B. Peck \& W.H. Whitcomb. 1967. Notes on spider parasites, including a reference list. Journal of the Kansas Entomological Society 40:422-434.

Eggleton, P. \& R. Belshaw. 1992. Insect parasitoids: an evolutionary overview. Philosophical Transactions of the Royal Society of London, Series B 337:1-20.
Eickstedt, V.R. 1971. Three cases of parasitism in the mygalomorph spider Lasiodora klugi (C. L. Koch) by a fly of the genus Exetasis (Diptera, Acroceridae) in Brazil. Memórias do Instituto Butantã 35:139-146.

Emerton, F.H. 1890. An internal dipterous parasite of spiders. Psyche 5:404.

Evans, R.E. 1969. Parasites of spiders and their eggs. Proceedings of the Birmingham Natural History Society 21:156-168.

Feener, D.H. \& B.V. Brown. 1997. Diptera as parasitoids. Annual Review of Entomology 42:73-97.

Fitton, M.G., M.R. Shaw \& A.D. Austin. 1987. The Hymenoptera associated with spiders in Europe. Zoological Journal of the Linnean Society 90:65-93.

Foote, B.A. 1984. Biology of Trimerina madizans, a predator of spider eggs (Diptera: Ephydridae). Proceedings of the Entomological Society of Washington 86:486-492.

Gagne, R.J. 1995. Revision of Tetranychid (Acarina) mite predators of the genus Feltiella (Diptera, Cecidomyiidae). Annals of the Entomological Society of America 88:16-30.

Gauld, I.D. \& J. Dubois. 2006. Phylogeny of the Polysphincta group of genera (Hymenoptera: Ichneumonidae; Pimplinae), a taxonomic revision of spider ectoparasitoids. Systematic Entomology 31:529 564.

Goldblatt, P., J.C. Manning \& P. Bernhardt 1997. Notes on the pollination of Gladiolus brevifolius (Iridaceae) by bees (Anthophoridae) and bee mimicking flies (Psilodera: Acroceridae). Journal of the Kansas Entomological Society 70:297-304

Goto, T. 1985. Megaselia (Aphiochaeta) araneivora sp. nov., an egg predator of the orb-web spider Argiope aemura (Walckenaer) in Japan (Diptera, Phoridae). Esakia 23:77-84.

Guarisco, H. 1990 A new spider host association for Ogcodes eugonatus (Loew) (Diptera, Acroceridae). Transactions of the Kansas Academy of Science 93:136-137.

Guarisco, H. 2001. Description of the egg sac of Mimetus notius (Araneae, Mimetidae) and a case of egg predation by Phalacrotophora epeirae (Diptera, Phoridae). Journal of Arachnology 29:267-269.

Hall, D.G. 1937. The North and Central American spider parasites of the genus Pseudogaurax (Diptera: Chloropidae). Journal of the Washington Academy of Sciences 27:255-261.

Hardy, D.E. 1965. Diptera: Cyclorrhapha II, Series Schizophora, Section Acalypteratae I. Family Drosophilidae. Insects of Hawaii, Volume 12. University of Hawaii Press, Honolulu.

Hieber, C.S. \& G.W. Uetz. 1990. Colony size and parasitoid load in two species of colonial Metepeira spiders from Mexico (Araneae: Araneidae). Oecologia 82:145-150.

Holl, A., T. Klett \& M. Kracht. 1983. Ogcodes fumatus (Erichson) (Diptera: Acroceridae) als parasit der Radnetzspinne Zygiella $x$ notata (Clerck). Hessische Faunistische Briefe 3:26-30.

Humphreys, W.F. 1976. The population dynamics of an Australian wolf spider, Geolycosa godeffroyi (L. Koch 1865) (Araneae: Lycosidae). Journal of Animal Ecology 45:59-80.

Jenks, G.E. 1938. Marvels of Metamorphosis. A scientific "G-Man" pursues rare trapdoor spider parasites for three years with a spade and a candid camera. National Geographic Magazine 75:807-828.

Jenks, G.E. 1940. The spider's "uninvited" fly brings doom. Natural History 45:157-161.

Johnson, C.W. 1915. Note on the species of the genus Acrocera. Psyche 22:198-203.

Jones, S.E. 1940. An annotated list of the spiders of an East Central Illinois forest (Wm. Trelease Woods, University of Illinois). Transactions of the Illinois Academy of Science 33:216-220.

Kanmiya, K. 1972. New record of a spider predator of the genus Pseudogaurax Malloch, 1915, from Japan, with a note on the European species Gaurax venustus Czerny (Diptera: Chloropidae). Mushi 46:39-43. 
Kanmiya, K. 1983. A systematic study of the Japanese Chloropidae. Memoirs of the Entomological Society of Washington 11:1-370.

Kaston, B.J. 1937. Notes on dipterous parasites of spiders. Journal of the New York Entomological Society 45:415-420.

Kaston, B.J. \& G.E. Jenks. 1937. Dipterous parasites of spider egg sacs. Bulletin of the Brooklyn Entomological Society 32:160-165.

Kehlmaier, C. \& J.M. Almeida. 2014. New host records for European Acroceridae (Diptera), with discussion of species limits of Acrocera orbiculus (Fabricius) based on DNA-barcoding. Zootaxa 3780:135-152.

Kehlmaier, C., R. Michalko \& S. Korenko. 2012. Ogcodes fumatus (Diptera: Acroceridae) reared from Philodromus cespitum (Araneae: Philodromidae), and first evidence of Wolbachia Alphaproteobacteria in Acroceridae. Annales Zoologici 62:281-286.

Kerr, P.H. \& S.L. Winterton. 2008. Do parasitic flies attack mites? Evidence in Baltic amber. Biological Journal of the Linnean Society 93:9-13.

King, J.L. 1916. Observations on the life history of Pterodontia flavipes Gray. Annals of the Entomological Society of America 9:309-321.

Kintner, E. 1935. Some spiders of the genus Tetragnatha. Proceedings of the Indiana Academy of Science 44:207-209.

Koch, C. 1872. Beitrag zur Dipterenfauna Tirols. Zeitschrift Des Ferdinandeums Für Tirol Und Vorarlberg 17:329-344.

Krehenwinkel, H., D. Rodder, M. Naparus-Aljancic \& M. Kuntner. 2016. Rapid genetic and ecological differentiation during the northern range expansion of the venomous yellow sac spider Cheiracanthium punctorium in Europe. Evolutionary Applications Online. doi:10.1111/eva.12392.

Krijger, J.P. 1910. Notes and observations on some parasites on spider's eggs. Entomologiske Meddelelser 8:257-258.

Lamore, D.H. 1960. Cases of parasitism of the basilica spider, Allepeira lemniscata (Walckenaer), by the dipteran endoparasite, Ogcodes dispar (Macquart). Proceedings of the Entomological Society of Washington 62:65-85.

Langer, G. 2005. Ein seltener Parasitoid der Wolfspinne Pardosa alacris (Araneae: Lycosidae): Ogcodes gibbosus (Diptera: Acroceridae). Arachnologischen Mitteilungen 29:45-48.

Lapoint, R.T., P.M. O'Grady \& N.K. Whiteman. 2013. Diversification and dispersal of the Hawaiian Drosophilidae: the evolution of Scaptomyza. Molecular Phylogenetics and Evolution 69:95-108.

Larrivée, M. \& C.J. Borkent. 2009. New spider host associations for three acrocerid fly species (Diptera, Acroceridae). Journal of Arachnology 37:241-242.

Locket, G.H. 1930. Note on the life-history of Oncodes pallipes Latr. (Dipt.). The Entomologist 63:241.

Lockley, T.C. \& O.P. Young. 1993. Survivability of overwintering Argiope aurantia (Araneidae) egg cases, with an annotated list of associated arthropods. Journal of Arachnology 21:50-54.

Manuel, R.L. 1984. The egg sac of Pityohyphantes costatus (Hentz) (Araneae, Linyphiidae) and its phorid parasite. Journal of Arachnology 12:371-372.

Marshall, S.A. 2012. Flies: The Natural History and Diversity of Diptera. Firefly Books, New York.

Marshall, S.A., A. Borkent, I. Agnarsson, G.W. Otis, L. Fraser \& D. d'Entremont. 2015. New observations on a neotropical termitehunting theridiid spider: opportunistic nest raiding, prey storage, and ceratopogonid kleptoparasites. Journal of Arachnology 43:419-421

McQueen, D.J. 1978. Field studies of growth, reproduction and mortality in the burrowing wolf spider Geolycosa domifex (Hancock). Canadian Journal of Zoology 56:2037-2049.

McQueen, D.J. 1983. Mortality patterns for a population of burrowing wolf spiders Geolycosa domifex (Hancock), living in southern Ontario. Canadian Journal of Zoology 61:2758-2767.
Melander, A.L. 1902. Notes on the Acroceridae. Entomological News 13:178-182.

Menge, A. 1866. Preussiche Spinnen, Part 4. A.W. Kafemann, Danzig.

Millot, J. 1938. Le développement et la biologie larvaire des Oncodides (= Cyrtidés), Diptères parasites d'araignées. Bulletin de la Société Zoologique de France 63:162-181.

Montgomery, T.H. 1903. Studies on the habitats of spiders particularly those of the mating period. Proceedings of the Academy of Natural Sciences of Philadelphia 55:59-149.

Moore, J. 2002. Parasites and the Behavior of Animals. Oxford University Press, Oxford.

Muma, M.H. \& K.J. Stone. 1971. Predation of Gasteracantha cancriformis (Arachnida: Araneidae) eggs in Florida citrus groves by Phalacrotophora epeirae (Insecta: Phoridae) and Arachnophaga ferruginea (Insecta: Eupelmidae). Florida Entomologist 54:305310.

Nartshuk, E.P. 1997. Family Acroceridae. Pp. 469-485. In Contributions to a Manual of Palaearctic Diptera (with Special Reference to Flies of Economic Importance), Volume 2, Nematocera and Lower Brachycera. (L. Papp, B. Darvas, eds.). Science Herald, Budapest.

Nielsen, E. 1932. The Biology of Spiders. With Especial Reference to the Danish Fauna. Levin \& Munksgaard, Copenhagen.

Noyes, J.S. 2016. Universal Chalcidoidea database. Online at http:// www.nhm.ac.uk/research-curation/research/projects/chalcidoids/

O'Grady, P., J. Bonacum, R. Desalle \& F. Val. 2003. The placement of Engiscaptomyza, Grimshawomyia, and Titanochaeta, three clades of endemic Hawaiian Drosophilidae (Diptera). Zootaxa 159:1-16.

Overgaard Nielsen, B., P. Funch \& S. Toft. 1999. Self-injection of a dipteran parasitoid into a spider. Naturwissenschaften 86:530-532.

Pape, T. 1986. Afrotropical species of Metopia (Insecta, Diptera, Sarcophagidae). Steenstrupia 124:73-84.

Pape, T. \& F.C. Thompson. 2013. Systema Dipterorum, Version 1.5. Online at http://www.diptera.org

Pape, T., G. Dahlem, C.A. Mello Patiu \& M. Giroux. 2012. The World of Flesh Flies (Diptera: Sarcophagidae). Online at http:// www.zmuc.dk/entoweb/sarcoweb/sarcweb/sarc_web.htm

Pichka, V.E. 1977. On insects parasitizing in spiders, their cocoons and nests. Vestnik Zoologii 3:92-93.

Pierce, W.D. 1942. Fauna and flora of the El Segundo sand dunes. 12. Utilization of the black widow parasite and further data on spiders and parasites. Bulletin of the Southern California Academy of Sciences 41:14-28.

Potgieter, C.J., T.J. Edwards, R.M. Miller \& J. Van Staden. 1999. Pollination of seven Plectranthus spp. (Lamiaceae) in southern Natal, South Africa. Plant Systematics and Evolution 218:99-112.

Prakash, R.N. \& T.J. Pandian. 1977. Energy flow from spider eggs through dipteran parasite and hymenopteran hyperparasite populations. Oecologia 33:209-219.

Price, P.W. 1980. The Evolutionary Biology of Parasites. Princeton University Press, Princeton, New Jersey.

Pujol-Luz, J.R. 2004. A associação de Philopota sp. Wiedemann (Diptera, Acroceridae) com flores do gervão-azul, Stachytarphetta cayenensis (Verbenaceae) na Ilha da Marambaia, Rio de Janeiro, Brasil. Entomología y Vectores 11:681-687.

Robinson, M.H. \& B. Robinson. 1977. Associations between flies and spiders: bibiocommensalism and dipsoparasitism? Psyche 84:150157.

Rollard C. 1984. Composition et structure de la biocénose consommatrice des Aranéides. Revue Arachnologique 5:211-237.

Sabrosky, C.W. 1948. A further contribution to the classification of North American spider parasites of the family Acroceridae. American Midland Naturalist 39:382-440.

Sabrosky, C.W. 1966. Three new Brazilian species of Pseudogaurax, with a synopsis of the genus in the Western Hemisphere (Diptera, 
Chloropidae). Papéis Avulsos do Departamento de Zoologia 19:117-127.

Sabrosky, C.W. 1990. New synonymy and new species in Neotropical Chloropidae (Diptera). Memórias do Instituto Oswaldo Cruz 84:463-465.

Schlinger, E.I. 1952. The emergence, feeding habits, and host of Opsebius diligens Osten Sacken. Pan-Pacific Entomologist 28:7-12.

Schlinger, E.I. 1960. A revision of the genus Ogcodes Latreille, with particular reference to species of the Western hemisphere. Proceedings of the United States National Museum 111:227-336.

Schlinger, E.I. 1968. Studies in neotropical Acroceridae, Part 1. A revision of Arrhynchus Philippi and a key to the genera of the Ocnaea branch of the Panopinae (Diptera). Revista Chilena De Entomología 6:47-54.

Schlinger, E.I. 1972. A new Brazilian panopine species, Exetasis eickstedtae, reared from the theraphosid spider, Lasiodora klugi (Koch), with a description of its immature larval stages (Diptera, Acroceridae). Papéis Avulsos de Zoologia 26:73-82.

Schlinger, E.I. 1981. Acroceridae. Pp. 575-584. In Manual of Nearctic Diptera. Volume I. (J.F. McAlpine, B.V. Peterson, G.E. Shewell, H.J. Teskey, J.R. Vockeroth \& D.E.M. Wood, eds.). Research Branch, Agriculture Canada, Ottawa.

Schlinger, E.I. 1987. The biology of Acroceridae (Diptera): true endoparasitoids of spiders. Pp. 319-327. In Ecophysiology of Spiders. (W. Nentwig, ed.). Springer-Verlag, Berlin.

Schlinger, E.I. 2003. The spider-endoparasitoids of Madagascar (Diptera: Acroceridae). Pp. 734-740. In The Natural History of Madagascar. (S.M. Goodman, J.P. Benstead, eds.). University of Chicago Press, Chicago \& London.

Schlinger, E.I., J.P. Gillung \& C.J. Borkent. 2013. New spider flies from the Neotropical Region (Diptera, Acroceridae) with a key to New World genera. Zookeys 270:59-93.

Schwendinger, P.J. \& T. Pape. 2000. Metopia sinensis (Diptera, Sarcophagidae), an unusual predator of Liphistius (Araneae, Mesothelae) in Northern Thailand. Journal of Arachnology 28:353-356.

Séguy, E. 1926. Faune de France, 13, Diptères (Brachyceres). Lechevalier, Paris.

Sferra, N.J. 1986. First record of Pterodontia flavipes (Diptera: Acroceridae) larvae in the mites Podothrombium (Acari: Trombidiidae) and Abrolophus (Acari: Erythraeidae). Entomological News 97:121-123.

Shi, C.M., Z. Xue-Shu \& Z. De-Xing. 2015. Parasitoidism of the Sarcophaga dux (Diptera: Sarcophagidae) on the Mesobuthus martensii (Scorpiones: Buthidae) and its implications. Annals of the Entomological Society of America 108:978-985.

Shinonaga, S. \& A.T. Barrion. 1980. A new species of sarcophagid fly parasitic in the egg of the spider, Argiope catenulata (Dolescall) in the Philippines. Kontyu, Tokyo 48:537-539.

Sivinski, J. \& M. Stowe. 1980. A kleptoparasitic cecidomyiid and other flies associated with spiders. Psyche 87:337-348.

Sivinski, J., S. Marshall \& E. Petersson. 1999. Kleptoparasitism and phoresy in the Diptera. Florida Entomologist 82:179-197.

Souza Lopes, H. 1985. New genus of Sarcophagidae (Diptera) based on an Australian species living on spider egg cases. Australian Entomologist 12:51-53.

Stireman, J.O., J.E. O'Hara \& D.M. Wood. 2006. Tachinidae: evolution, behavior, and ecology. Annual Review of Entomology 51:525-555.

Tewksbury, J.J., J.G.T. Anderson, J.D. Bakker, T.J. Billo, P.W. Dunwiddie, M.J. Groom et al. 2014. Natural history's place in science and society. BioScience 64:300-310.

Toft, S., B. Overgaard Nielsen \& P. Funch. 2012. Parasitoid suppression and life-history modifications in a wolf spider following infection by larvae of an acrocerid fly. Journal of Arachnology 40:13-17.
Townsend, C.H.T. 1893. A scorpion parasite. Journal of the Institute of Jamaica 1:221.

Trojan, P. 1956. Oncodes reginae sp. n. and notes on the European species of the family Cyrtidae. Annales Zoologici 16:73-79.

Vachon, M. 1952. Remarques préliminaires sur quelques insectes parasites (Diptères et Hyménoptères) des cocons et œufs d'Araignées. Bulletin du Muséum National d'histoire Naturelle 24:200 203.

van Helsdingen, P.J. 2011. Spiders in a hostile world (Arachnoidea, Araneae). Arachnologische Mitteilungen 40:55-64.

Vellard, J. 1934. Notes sur quelques parasites de Mygales SudAmericaines. Bulletin de la Société Zoologique de France 59:293295.

Vetter, R.S., L.S. Vincent, A.A. Itnyre, D.E. Clarke, K.I. Reinker, D.W.R. Danielsen et al. 2012. Predators and parasitoids of egg sacs of the widow spiders, Latrodectus geometricus and Latrodectus hesperus (Araneae: Theridiidae) in southern California. Journal of Arachnology 40:209-214.

Vincent, L.S. 1985. The first record of a tachinid fly as an internal parasitoid of a spider (Diptera: Tachinidae; Araneae: Antrodiaetidae). Pan-Pacific Entomologist 61:224-225.

Vincent, L.S. 1986. Pathogens and parasitoids of the fossorial mygalomorph spider, Atypoides riversi O. P. Cambridge (Antrodiaetidae: Araneae) of various age and size classes. Pp. 291-294. In Proceedings of the IX International Congress of Arachnology, Panama 1983. (W.G. Eberhard, Y.D. Lubin, B.C. Robinson, eds.). Smithsonian Institution Press, Washington.

Viquez, C. \& L.F. De Armas. 2009. Parasitism on whip spider eggs (Arachnida: Amblypygi) by Chloropidae flies (Insecta: Diptera). Boletin de la SEA 45:541-542.

Wandolleck, B. 1906. A new species of Cyrtidae from North Queensland bred by F. P. Dodd. Transactions of the Entomological Society of London 54:131-132.

Weinmann, D. \& R.H.L. Disney. 1997. Two new species of Phoridae (Diptera) whose larvae associate with large spiders (Araneae: Theraphosidae). Journal of Zoology 243:319-328.

Williams, S.C., P.H. Arnaud Jr. \& G. Lowe. 1994. Parasitism of Anuroctonus phaiodactylus (Wood) and Vaejovis spinigerus (Wood) (Scorpiones: Vaejovidae) by Spilochaetosoma californicum Smith (Diptera: Tachinidae), and a review of parasitism in Scorpiones. Myia 5:11-27.

Winterton, S.L., B.M. Wiegmann \& E.I. Schlinger. 2007. Phylogeny and Bayesian divergence time estimations of small-headed flies (Diptera: Acroceridae) using multiple molecular markers. Molecular Phylogenetics and Evolution 43:808-832.

Wirth, W.W. 1952. Two new spider egg predators from the Hawaiian Islands (Diptera: Drosophilidae). Proceedings of the Hawaiian Entomological Society 14:415-417.

Wirth, W.W., W.N. Mathis \& J.R. Vockeroth. 1987. Ephydridae. Pp. 1027-1048. In Manual of Nearctic Diptera. Volume I. (J.F. McAlpine, B.V. Peterson, G.E. Shewell, H.J. Teskey, J.R. Vockeroth, D.E.M. Wood, eds.). Research Branch, Agriculture Canada, Ottawa.

World Spider Catalog. 2016. World Spider Catalog. Version 17.5. Natural History Museum, Bern. Online at http://wsc.nmbe.ch

Yeates, D.K. \& D. Greathead. 1997. The evolutionary pattern of host use in the Bombyliidae (Diptera): a diverse family of parasitoid flies. Biological Journal of the Linnean Society 60:149-185.

Zaitsev, V.F. \& D. Charykuliev. 1981. On the biology of bee-flies of the genus Petrorossia Bezzi (Diptera, Bombyliidae) with description of a new species from Turkmenia. Entomological Review 60:158-160.

Manuscript received 22 November 2016, revised 2 December 2016. 


\section{$2 \mathrm{BHL}$ Biodiversity Heritage Library}

Gillung, Jéssica P and Borkent, Christopher J. 2017. "Death comes on two wings: a review of dipteran natural enemies of arachnids." The Journal of arachnology 45(1), 1-19. https://doi.org/10.1636/joa-s-16-085.1.

View This Item Online: https://www.biodiversitylibrary.org/item/274714

DOI: https://doi.org/10.1636/joa-s-16-085.1

Permalink: https://www.biodiversitylibrary.org/partpdf/289888

\section{Holding Institution}

Smithsonian Libraries

\section{Sponsored by}

Biodiversity Heritage Library

\section{Copyright \& Reuse}

Copyright Status: In Copyright. Digitized with the permission of the rights holder

Rights Holder: American Arachnological Society

License: https://creativecommons.org/licenses/by-nc-sa/4.0/

Rights: http://www.biodiversitylibrary.org/permissions/

This document was created from content at the Biodiversity Heritage Library, the world's largest open access digital library for biodiversity literature and archives. Visit BHL at https://www.biodiversitylibrary.org. 